\title{
Circadian clock component REV-ERB $\alpha$ controls homeostatic regulation of pulmonary inflammation
}

\author{
Marie Pariollaud, ${ }^{1}$ Julie E. Gibbs, ${ }^{1}$ Thomas W. Hopwood, ${ }^{1}$ Sheila Brown, ${ }^{1}$ Nicola Begley, ${ }^{1}$ Ryan Vonslow, ${ }^{1}$ Toryn Poolman, \\ Baoqiang Guo, ${ }^{1}$ Ben Saer, ${ }^{1}$ D. Heulyn Jones, ${ }^{2}$ James P. Tellam, ${ }^{2}$ Stefano Bresciani, ${ }^{2}$ Nicholas C.0. Tomkinson, ${ }^{2}$ \\ Justyna Wojno-Picon, ${ }^{2,3}$ Anthony W.J. Cooper, ${ }^{2,3}$ Dion A. Daniels, ${ }^{3}$ Ryan P. Trump, ${ }^{4}$ Daniel Grant, ${ }^{4,5}$ William Zuercher, ${ }^{4,6}$ \\ Timothy M. Willson, ${ }^{4,6}$ Andrew S. MacDonald, ${ }^{1}$ Brian Bolognese, ${ }^{7}$ Patricia L. Podolin, ${ }^{7}$ Yolanda Sanchez, ${ }^{7}$ \\ Andrew S.I. Loudon, ${ }^{1}$ and David W. Ray ${ }^{1}$ \\ ${ }^{1}$ Faculty of Biology, Medicine and Health, University of Manchester, Manchester, United Kingdom. ${ }^{2}$ Department of Pure and Applied Chemistry, University of Strathclyde, Glasgow, United Kingdom. \\ ${ }^{3}$ GlaxoSmithKline R\&D, Stevenage, United Kingdom. ${ }^{4}$ Molecular Discovery Research, ClaxoSmithKline, Research Triangle Park, North Carolina, USA. ${ }^{5}$ Novartis AG, East Hannover, New Jersey, USA. \\ ${ }^{6}$ Eshelman School of Pharmacy, University of North Carolina at Chapel Hill, Chapel Hill, North Carolina, USA. ${ }^{7}$ Stress and Repair Discovery Performance Unit, Respiratory Therapy Area, \\ GlaxoSmithKline, King of Prussia, Pennsylvania, USA.
}

\begin{abstract}
Recent studies reveal that airway epithelial cells are critical pulmonary circadian pacemaker cells, mediating rhythmic inflammatory responses. Using mouse models, we now identify the rhythmic circadian repressor REV-ERB $\alpha$ as essential to the mechanism coupling the pulmonary clock to innate immunity, involving both myeloid and bronchial epithelial cells in temporal gating and determining amplitude of response to inhaled endotoxin. Dual mutation of REV-ERB $\alpha$ and its paralog REV-ERB $\beta$ in bronchial epithelia further augmented inflammatory responses and chemokine activation, but also initiated a basal inflammatory state, revealing a critical homeostatic role for REV-ERB proteins in the suppression of the endogenous proinflammatory mechanism in unchallenged cells. However, REV-ERB $\alpha$ plays the dominant role, as deletion of REV-ERB $\beta$ alone had no impact on inflammatory responses. In turn, inflammatory challenges cause striking changes in stability and degradation of REV-ERB $\alpha$ protein, driven by SUMOylation and ubiquitination. We developed a novel selective oxazole-based inverse agonist of REV-ERB, which protects REV-ERB $\alpha$ protein from degradation, and used this to reveal how proinflammatory cytokines trigger rapid degradation of REV-ERB $\alpha$ in the elaboration of an inflammatory response. Thus, dynamic changes in stability of REV-ERB $\alpha$ protein couple the core clock to innate immunity.
\end{abstract}

\section{Introduction}

Protection of pulmonary mucosal surfaces from infection requires complex interplay between the airway epithelial cells and mononuclear phagocyte populations both resident within the lung (1). Airway macrophages function as required accessory cells for optimal lung performance, with adverse consequences arising from either insufficient or excessive activation. Bronchial epithelial cells maintain an intact mucosal barrier, and transmit inhibitory signals to airway macrophages, maintaining homeostatic control of airway inflammation. In response to bacterial infection, neutrophil recruitment into the inflamed lung from the circulating neutrophil compartment is a necessary and closely regulated phenomenon, with avoidable tissue damage the consequence for excessive neutrophil infiltration. The determinants and controls maintaining pulmonary immune homeostasis and regulating inflammatory responses remain poorly defined. Recently, we discovered that the

Conflict of interest: The authors have declared that no conflict of interest exists. License: This work is licensed under the Creative Commons Attribution 4.0 International License. To view a copy of this license, visit http://creativecommons.org/ licenses/by/4.0/

Submitted: March 9, 2017; Accepted: March 8, 2018

Reference information: J Clin Invest. 2018;128(6):2281-2296.

https://doi.org/10.1172/JCI93910. circadian clock exerted a major impact on the airway inflammatory response to challenge (2).

The central clock lies in the suprachiasmatic nucleus of the brain and maintains essential synchrony of peripheral tissue clocks via neural and humoral mediators. Virtually all cells in the body express components of the cellular circadian clock and are capable of sustaining circadian oscillations. This cellular circadian oscillator consists of a positive arm - CLOCK and BMAL1 heterodimers - driving transcription of 2 inhibitory arms - PER/ CRY and REV-ERB $\alpha /$ REV-ERB $\beta$ (also known as NR1D1 and NR1D2), which feed back to inhibit BMAL1/CLOCK heterodimer transactivation function (3). The circadian clock powerfully regulates inflammation (4-6). Multiple measures of innate immunity show strong time-of-day variation, including cell trafficking to tissues, and monocyte/macrophage responses to TLR activation $(7,8)$, but far less is known about circadian control of inflammation in an organ context, such as the lung. Previously, we identified a strong time-of-day variation in pulmonary inflammation, and response to pneumococcal infection, a prevalent infectious challenge to the lung (2). This study showed that circadian clock disruption by deletion of the essential clock gene BMAL1 in the myelomonocytic cells had little impact, but that BMAL1 deletion in the airway epithelial cells, driven by CCSP-iCre transgene, augmented inflammation across the circadian cycle. The circadian 
A

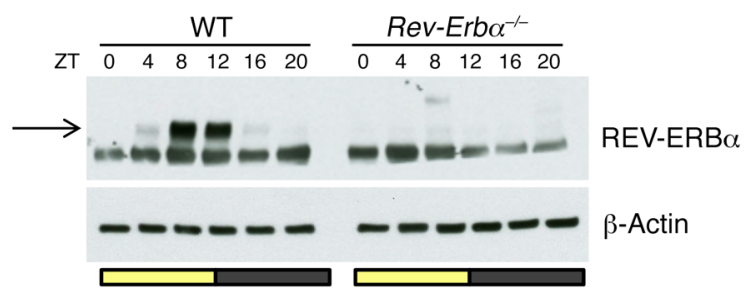

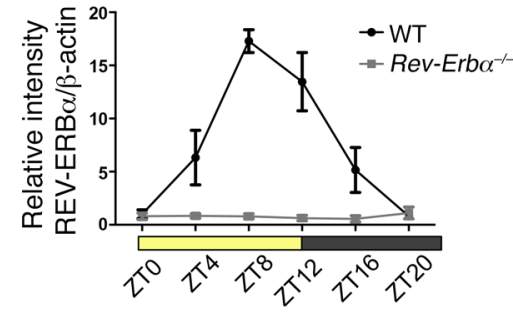
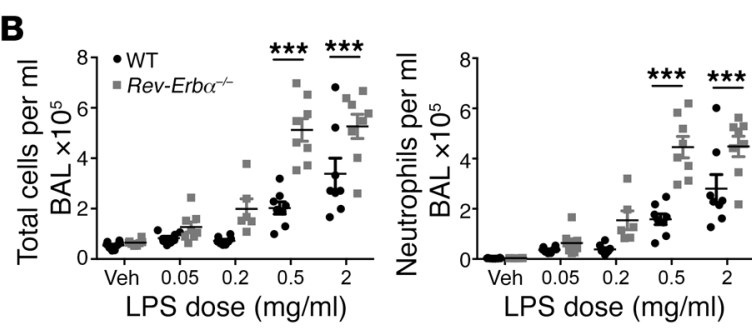
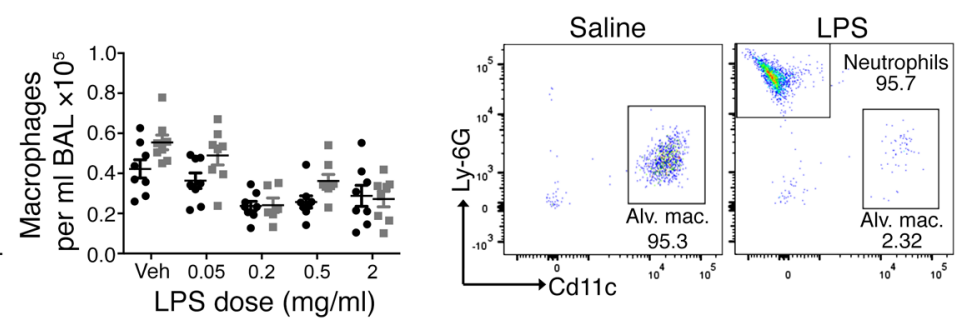

C

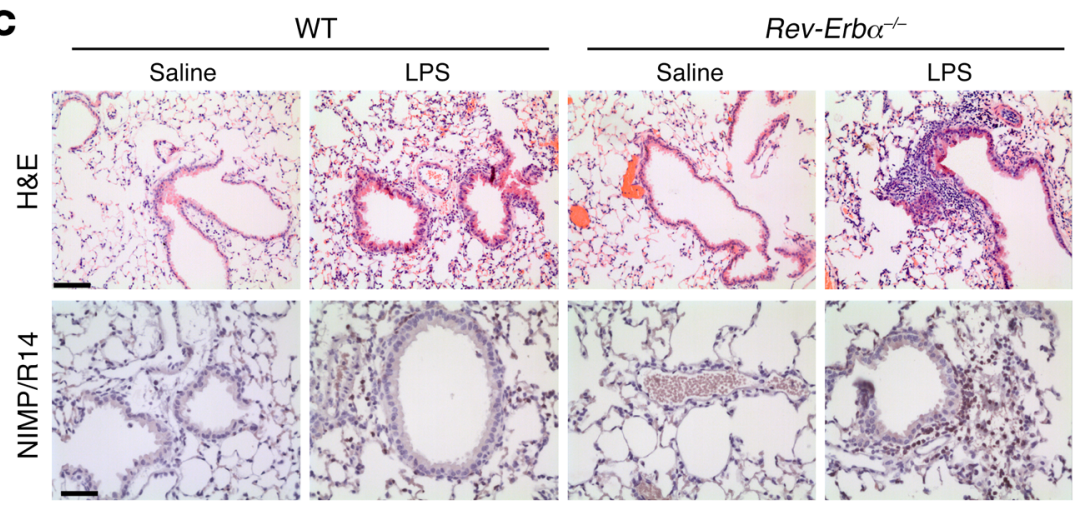

D

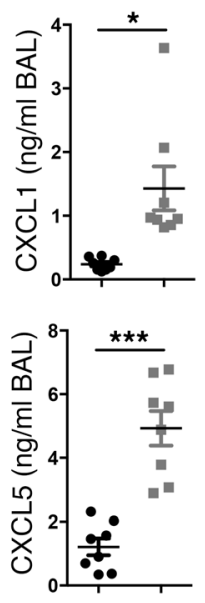

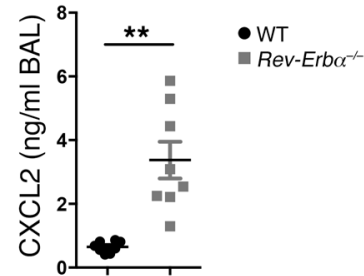

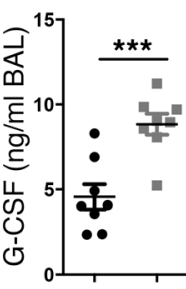

E
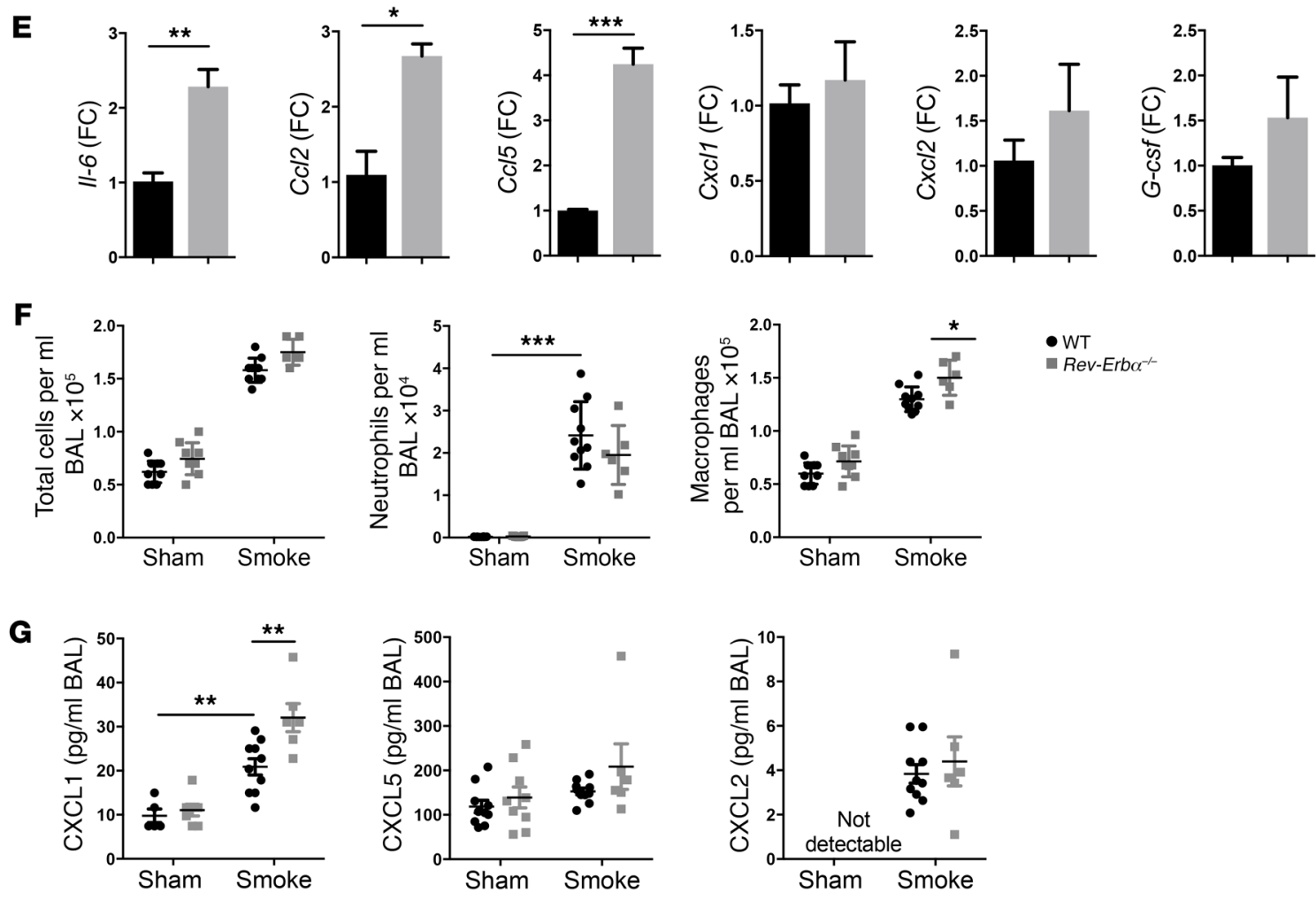
Figure 1. REV-ERB $\alpha$ plays a critical role in regulation of lung inflammation. (A) Whole-lung REV-ERB $\alpha$ protein across the day (ZT, time from lights on). REV-ERB $\alpha$ densitometry (mean $\pm \mathrm{SEM}$ ) was normalized to $\beta$-actin and to WT at ZTO; $n=5$ for WT and $n=3$ for Rev-Erbo $\alpha^{-/-}$per time point. (B) Mice were exposed to aerosolized LPS at ZT4 and culled 5 hours later; cellular infiltrates were quantified in BAL using flow cytometry. Data presented as mean \pm SEM; $n=6-8,{ }^{* *} P<0.001$ (2-way ANOVA, post hoc Bonferroni). Veh, vehicle. (C) H\&E staining and immunohistochemistry for the neutrophil maker (NIMP/R14) of lung sections from mice after LPS challenge at $2 \mathrm{mg} / \mathrm{ml}$. Representative of $n=4$; scale bars: $50 \mu \mathrm{m}$. (D) Cytokine/chemokine levels in BAL fluid from mice exposed to aerosolized LPS ( $2 \mathrm{mg} / \mathrm{ml})$. Representative of $n=8$, Student's $t$ test with Welch's correction. (E) Quantitative PCR ( $\mathrm{PPCR}$ ) analysis of cytokine transcripts in alveolar macrophages isolated from mice and stimulated ex vivo with LPS at $100 \mathrm{ng} / \mathrm{ml}$ for 2 hours. Data normalized to WT and presented as mean \pm SEM; $n=3,{ }^{*} P<0.05,{ }^{* *} P<0.01,{ }^{* * *} P<0.001$ (Student's $t$ test). FC, fold change. (F) Ten-day cigarette smoke exposures were performed between ZT8 and ZT10, and animals were culled 20 hours after the last exposure. Cellular infiltrates were quantified in BAL using a hemocytometer for total cell number and cytospin for neutrophil and macrophage counts. Data presented as mean \pm SEM; $n=6-10,{ }^{*} P<0.05$, ${ }^{* *} P<0.001$ (2-way ANOVA, post hoc Bonferroni). (G) Chemokine levels in BAL fluid after 10-day cigarette smoke exposures. Data presented as mean $\pm \mathrm{SEM} ; n=6-10,{ }^{* *} P$ $<0.01$ (2-way ANOVA, post hoc Bonferroni).

effect in epithelial cells was explained by aberrant regulation of a single dominant neutrophil chemokine, CXCL5.

BMAL1 plays many roles in addition to being a core circadian clock component. The immune regulatory functions of BMAL1 have been proposed to lie with regulation of the downstream REV-ERB transcription factors (5), CLOCK regulation of NF-KB function (9), and microRNA regulation (10). BMAL1 is not tractable to drug therapy; therefore, identifying how BMAL1 regulates lung inflammation is important to realize benefits in the clinic. The orphan nuclear receptor REV-ERB $\alpha$ has been shown to be an important intermediary molecular link between the core clock and inflammatory pathways in macrophages (5), possibly mediated by direct DNA binding to the promoters of proinflammatory chemokines (11), but also involving inhibition of distal proinflammatory cytokine enhancers selected by macrophage-lineagedetermining factors (12).

New insights into REV-ERB biology have resulted from lossof-function studies, and application of chemical biology tools to manipulate function $(5,13-15)$. These include the discovery that REV-ERB $\alpha$ and $\beta$ are functionally redundant in terms of maintaining circadian oscillations, with only a minor change in period length seen in REV-ERB $\alpha$-null mice $(13,16)$. However, a fascinating discovery was the functional dissociation between the DNA-binding domain (DBD) functions of REV-ERB $\alpha$, required for circadian clock regulation, and the DBD-independent functions, necessary for regulation of rhythmic hepatic lipid metabolism. This serendipitous finding resulted from a failed loxP gene targeting approach, which deleted the DBD as an in-frame cassette, resulting in expression of a hypomorph allele, REV-ERB $\alpha$ $\mathrm{DBD}^{\mathrm{m}}(14)$. This striking finding revealed that only a minority of the REV-ERB $\alpha$ target gene spectrum was affected by loss of the DBD, and that REV-ERB recruitment to DNA elements was driven in large part by the cell lineage-determining transcription factors HNF4A and HNF6 in the liver. REV-ERB $\alpha$, as a nuclear receptor, is a tractable drug target, with synthetic ligands regulating core clock function, macrophage activation, and energy metabolism $(5,17)$. Lack of potency coupled with low in vivo efficacy has stalled further development for REV-ERB ligands as therapeutics for human application; however, these molecules offer invaluable tools to dissect REV-ERB function.

Here we explore the hypothesis that REV-ERB $\alpha$ within the airway epithelial cells, and the sentinel macrophages, plays a critical role in pulmonary inflammation, and that REV-ERB $\alpha$ serves as a signaling relay for 2-way communication between the core circadian clock and elaboration of the inflammatory reaction. We identify distinct roles for REV-ERB $\alpha$ within bronchial epithelial cells and myelomonocytic cells in setting the amplitude and timing of neutrophilic inflammation in the lung. We find that REV-ERB $\alpha$ is dominant over REV-ERB $\beta$, but that there is some redundancy between the 2 paralogs. We identify a pathway linking inflammatory cytokine signaling through phosphorylation to SUMOylation, ubiquitination, and degradation of REV-ERB $\alpha$ protein, thereby relieving inhibition of the inflammatory response. We identify and test a new REV-ERB inverse agonist, which acts to inhibit inflammatory signaling, in part by stabilizing REV-ERB $\alpha$ protein, rendering it resistant to SUMO and ubiquitin modification. Thus, the clock/ immune interface is susceptible to pharmacological intervention.

\section{Results}

$R E V$-ERBa plays a critical role in regulation of lung inflammation. Previously, we have shown that BMAL1 deletion in the bronchial epithelium blocks circadian transcriptional cycles specifically in these cells, impacts on oxidative stress responses, suppresses both REV-ERB $\alpha$ and $\beta$ expression, and both abolishes time-of-day variation and augments pulmonary neutrophilic inflammation (2). Conditional deletion of BMAL1 is widely used in experimental models, as this is the only core molecular element driving circadian cycles in which suppression of function leads to abolition of rhythmic transcriptional oscillations in the cell. In order to identify specific circadian-regulated proteins that directly couple to the regulation of immunity, we focused on the circadian REV-ERB transcription factors, which have been previously been identified as strong candidates for the regulation of diverse physiological functions, including rhythmic regulation of hepatic metabolism and adipose tissue $(14,18)$. To identify the role of REV-ERB $\alpha$ in circadian control of lung inflammation, we first raised a monoclonal antibody that specifically detects REV-ERB $\alpha$ but not its paralog REV-ERB $\beta$ (Supplemental Figure 1; supplemental material available online with this article; https://doi.org/10.1172/ JCI93910DS1). This antibody revealed a marked daily pattern of REV-ERB $\alpha$ protein expression in whole lung, with a peak in the day around zeitgeber time ZT8-12 for WT mice, and absence of expression in knockout mice (Figure 1A). By convention, ZT0 is the time of lights-on. Protein expression was in close phase to the transcript rhythms, with peak expression between ZT8 and ZT12, suggesting a rapid transcriptional-translation process and turnover of the protein (Supplemental Figure 2A).

We tested responses of global REV-ERB $\alpha$ knockout (hereafter defined as Rev-Erbo-/-) and WT mice to nebulized lipopolysaccharide (LPS) at ZT4, when REV-ERB $\alpha$ protein is accumulating, and harvested animals 5 hours later, at peak of REV-ERB $\alpha$. We observed an exaggerated neutrophilic inflammation in knockout 
A

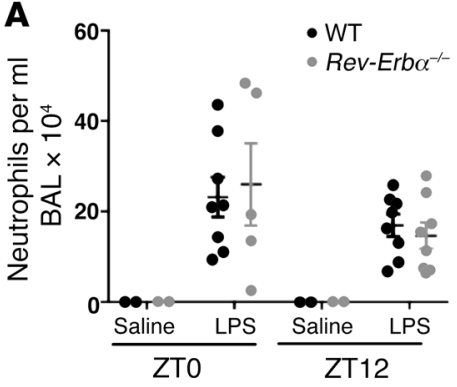

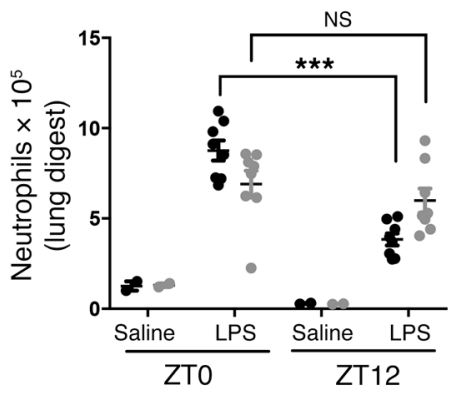

B

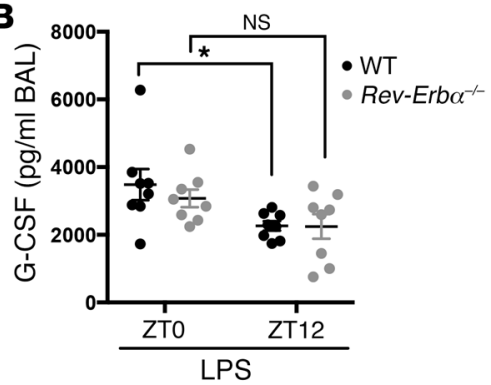

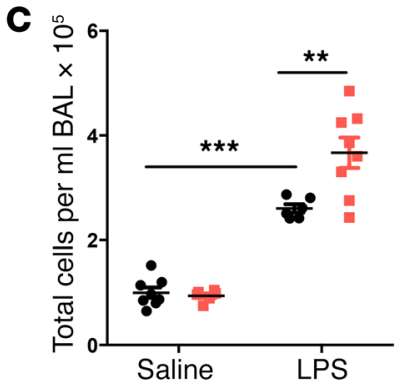
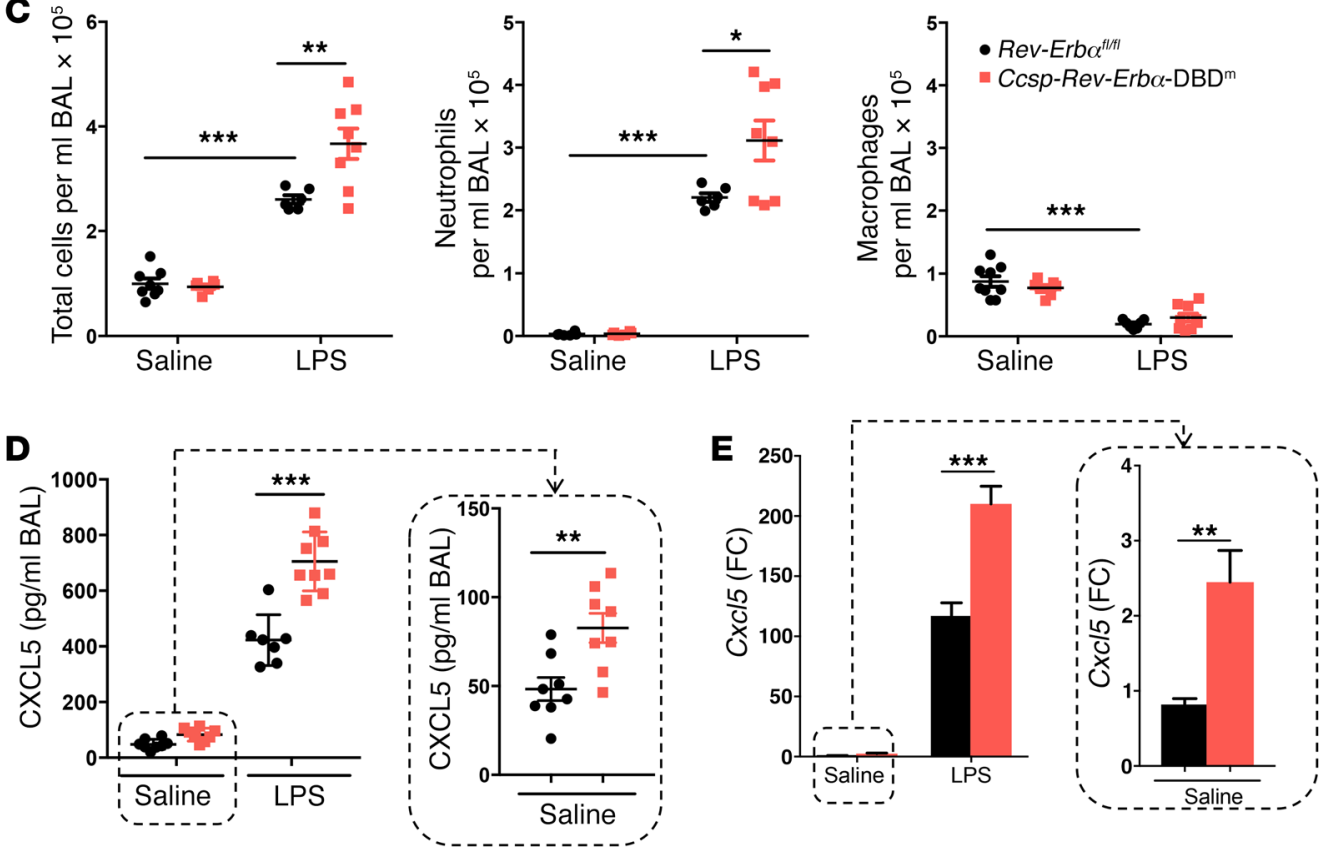

Figure 2. REV-ERB $\alpha$ in myeloid and airway epithelium regulates pulmonary inflammation. (A and B) Bone marrow cells from Rev-Erb $\alpha^{-/-}$or littermate controls were transplanted into WT recipient mice, which were then exposed to aerosolized LPS at $2 \mathrm{mg} / \mathrm{ml}$ or saline at indicated times for 20 minutes. (A) Neutrophil numbers in BAL samples or lung digests collected 5 hours after challenge, determined by flow cytometry analyses. (B) Chemokine protein levels in BAL samples. Data presented as mean $\pm \mathrm{SEM} ; n=2$ (saline) or 5-8 (LPS), ${ }^{*} P<0.05,{ }^{* * *} P<0.001$ (2-way ANOVA, post hoc Bonferroni). (C-E) Ccsp-RevErb $\alpha-\mathrm{DBD}^{\mathrm{m}}$ and littermate control mice were exposed to aerosolized LPS at $2 \mathrm{mg} / \mathrm{ml}$ or saline at ZT4 for 20 minutes. (C) Total cell counts in BAL samples collected 5 hours after challenge. Neutrophil and macrophage numbers in the same samples were determined by flow cytometry analyses. (D) Chemokine protein levels in BAL samples, measured using multiplex assay. (E) qPCR analysis of CxcI5 mRNA in lung tissues. Data normalized to saline Rev-Erba ${ }^{f / f l}$ control group. Data presented as mean \pm SEM; $n=5-9,{ }^{*} P<0.05,{ }^{* *} P<0.01,{ }^{* *} P<0.001$ (2-way ANOVA, post hoc Bonferroni).

mice (Figure 1, B and C), accompanied by significantly augmented chemokine and inflammatory cytokine responses, including CXCL5, the chemokine required to mediate the BMAL1 effect (ref. 2, Figure 1D, and Supplemental Table 1).

We have previously shown exaggerated pulmonary inflammatory responses at ZTO compared with ZT12 (2). Therefore, we repeated the challenges at the anticipated peak and trough of this natural inflammatory response oscillation (ZT0 and ZT12). These studies again revealed an increase in neutrophilic inflammation in WT mice at ZTO versus ZT12, but Rev-Erbo/- mice showed augmented responses at both time points, and loss of diurnal variation (Supplemental Figure 2, B and C, and Supplemental Table 2). The most striking genotype difference was seen at ZT12, and at that time point the knockout mouse bronchoalveolar lavage (BAL) chemokines CXCL1, CXCL2, and G-CSF were elevated, but we did not see any difference in CXCL5 (Supple- mental Table 2 and Supplemental Figure 2C), indicating a broader spectrum of REV-ERB $\alpha$ activity.

To extend these observations, we isolated alveolar macrophages from Rev-Erbo ${ }^{-/}$mice and showed that these exhibited increased ex vivo cytokine responses to LPS, including Il-6 (Figure 1E). However, we did not observe significant changes in Cxcl1 and Cxcl2 chemokines, while for $\mathrm{Cxcl} 5$ the primary source in pulmonary tissues is the epithelium and not alveolar macrophages (19).

We extended these observations to other innate inflammatory stimuli and assessed responses to a single exposure of cigarette smoke, a prevalent real-world environmental challenge to the lung. No cellular inflammatory responses were detected following this transient challenge, but $C x c l 5$ transcript was significantly elevated specifically in lungs of Rev-Erbo ${ }^{-/}$mice (Supplemental Figure 2, D and E). Following multiple smoke exposures (10 days), there was mortality of 3 of the 9 Rev-Erb $\alpha^{-/-}$mice, but none of the 
A

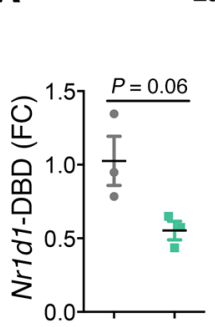

C

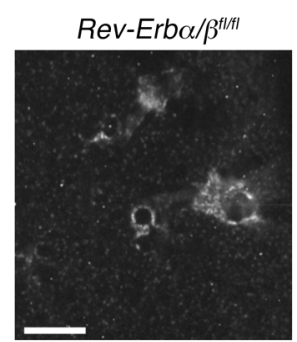

Bronchioles

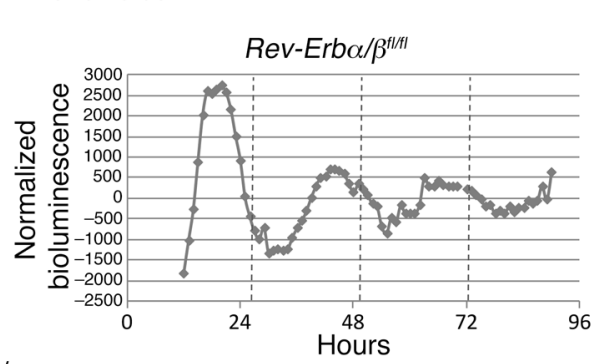

Ccsp-Rev-Erbo-DBD $/$

Rev-Erb $\beta^{-1-}$
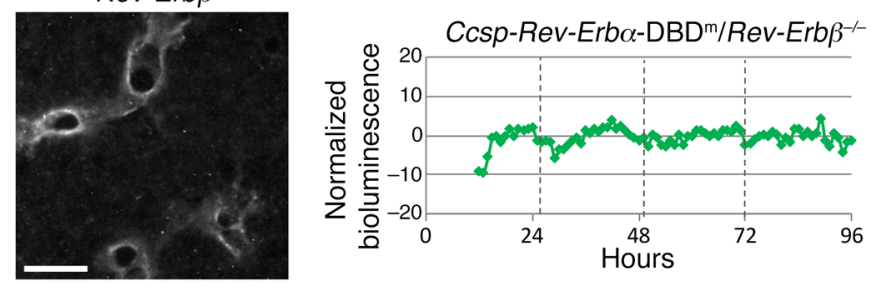

B

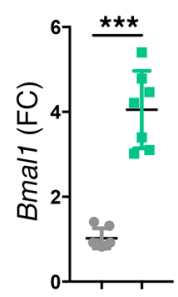

Whole lung

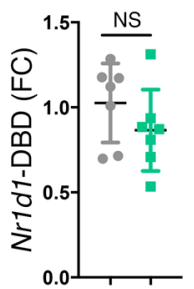

D
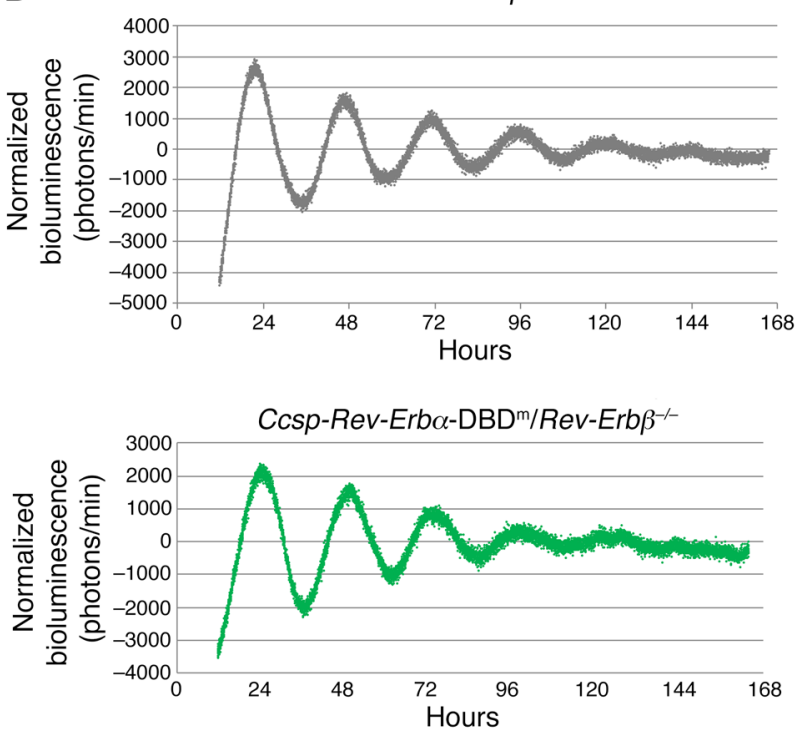

Figure 3. Both REV-ERB paralogs are required for circadian rhythms in the airway epithelium. (A) qPCR analysis of mRNA in bronchial epithelial cells, laser-captured from lung tissues collected at ZT9. Data normalized to Rev-Erb $\alpha / \beta^{f l / f l}$ control group and presented as mean $\pm \mathrm{SD} ; n=3$, ${ }^{* *} P<0.01, \mathrm{Stu}-$ dent's $t$ test. (B) qPCR analysis of mRNA in whole lung collected at ZTg. Data normalized to Rev-Erb $\alpha / \beta^{f l / f l}$ control group and presented as mean \pm SEM; $n=7$, ${ }^{* *} P<0.001$, Student's $t$ test. (C) Snapshots of PER2 oscillations in bronchioles within precision-cut lung slices. Scale bars: $500 \mu \mathrm{m}$. Bioluminescence intensity from bronchioles was quantified, normalized to a 24-hour moving average. Traces are representative of 2 biological replicates. (D) Bioluminescence recordings of whole-lung PER2 oscillations in precision-cut lung slices. Photon counts per minute were normalized to a 24-hour moving average, and traces are representative of 3 biological replicates.

WTs (nonsignificant by $\chi^{2}$ test). A slight increase in airway macrophages was observed in $\mathrm{Rev}-E \mathrm{~Eb} \alpha^{-/-}$mice compared with littermate controls (Figure 1F). There was an increase in CXCL1 in BAL samples from Rev-Erbo ${ }^{-/}$mice exposed to cigarette smoke compared with littermate controls, but no differences were observed for other chemokines and cytokines in BAL samples (Figure 1G), indicating a striking specificity of response.

Because of the lack of a robust cigarette smoke phenotype, and the identification of differentially regulated eosinophil chemokines (Supplemental Table 1; IL-5, CCL11), we analyzed an allergic inflammatory challenge using ovalbumin (OVA; Supplemental Figure 3 and ref. 20). There were no significant differences in response between genotypes, suggesting that REV-ERB $\alpha$ had particular selectivity for innate rather than adaptive immune responses in the lung.

Targeting Rev-Erbo in myeloid cells impairs time-of-day variation in pulmonary neutrophilic inflammation. Both alveolar macrophages and the airway epithelium provide the first lines of defense against respiratory pathogens $(21,22)$. To test the role of Rev-Erb $\alpha$ in the myeloid lineage, we performed bone marrow transplant studies from global Rev-Erb $\alpha$-null mice and from WT littermate controls (Figure 2, A and B, and Supplemental Figure 4A). Recipient mice showed only very minor changes in the circulating myeloid cell pool (Supplemental Figure 4A), but we noted that the time-of-day variation in pulmonary neutrophilia in the lung digests and in BAL G-CSF was not significant in myeloid Rev-Erb $\alpha_{-}$ null animals (Figure 2, A and B). In this study, we directly compared the cell yields from BAL and lung digestion, as we were concerned about the variance seen in some BAL experiments (Figure 2A, left panel). Lung digestion gives more precise measurements, but excludes other measurements from the lung tissue, and for that reason we returned to BAL measures for subsequent analyses.

We next generated mice with conditional deletion of the DBD of Rev-Erb $\alpha$ in myeloid-lineage cells by crossing floxed (Rev-Erbo $\alpha^{f / f l}$ ) mice (14) with a $L y s M^{\text {Cre/+ }}$ strain ( $\left.L y s M-R e v-E r b \alpha-D_{B D D}{ }^{m}\right)$ on a background of PER2::Luc in order to record circadian oscillations by bioluminescent recording. In this model, the protein retains other functions but is unable to bind directly to classical REV-ERB DNA response elements, and thereby loses its circadian clock regulating properties (14). Peritoneal macrophages derived from these 
A

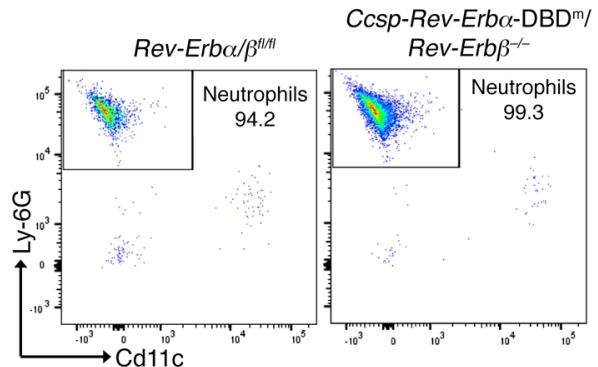

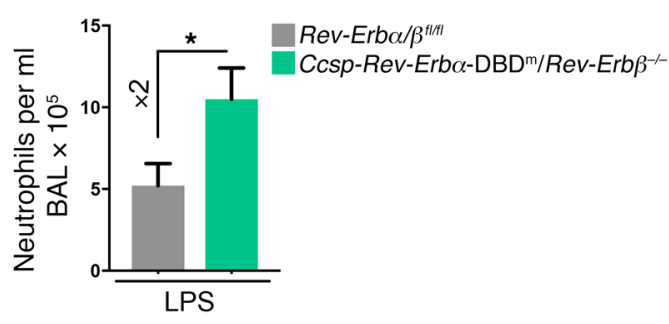
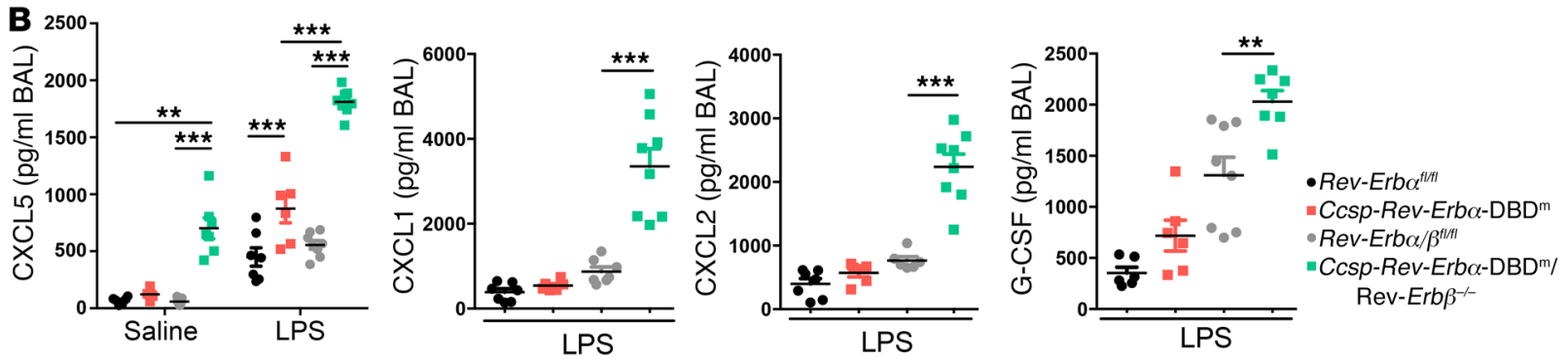

C
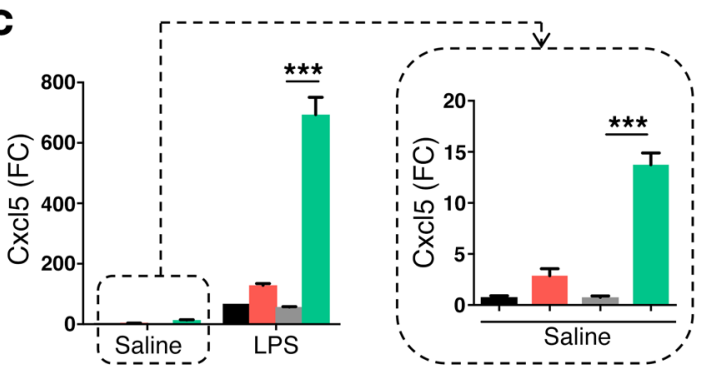

D
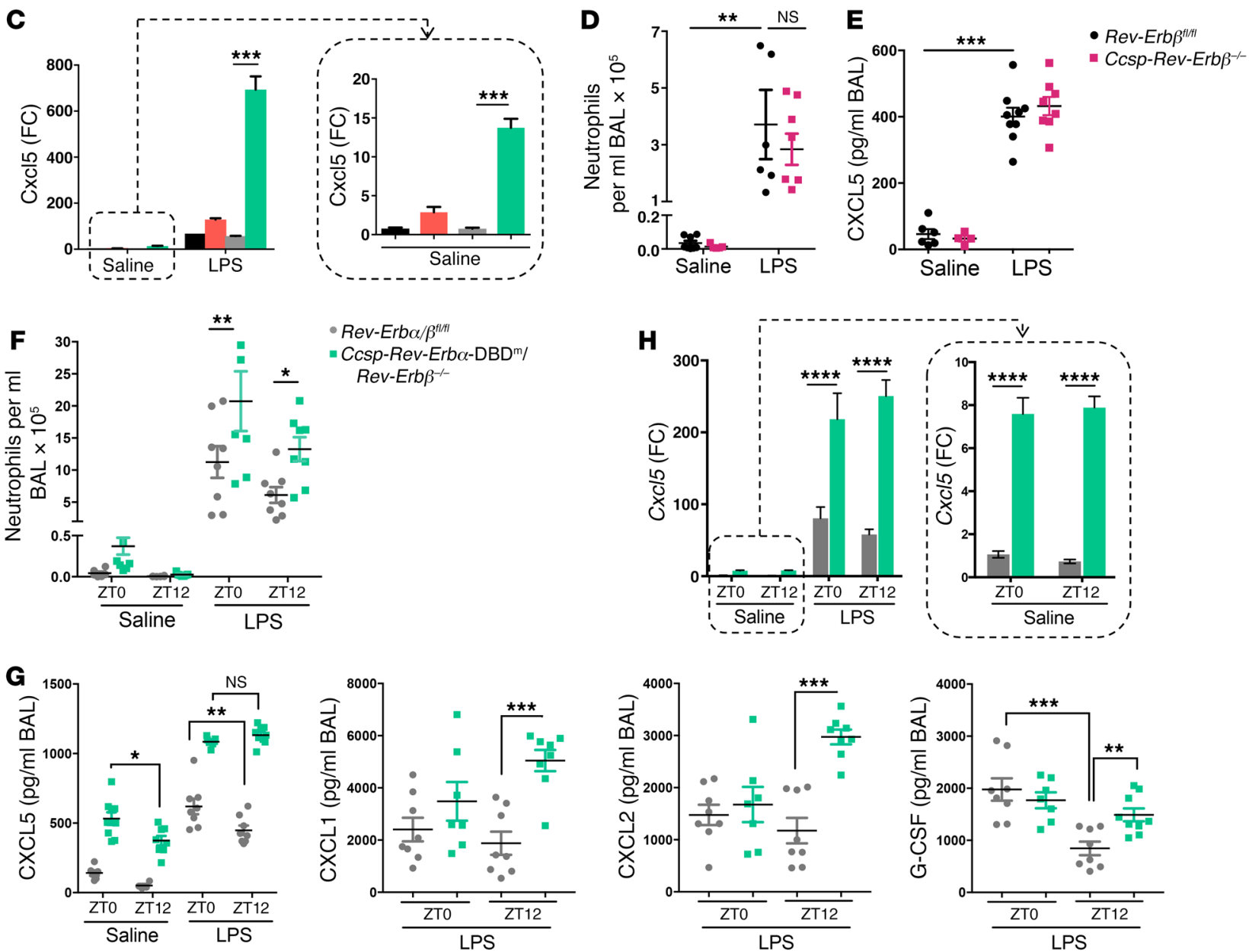

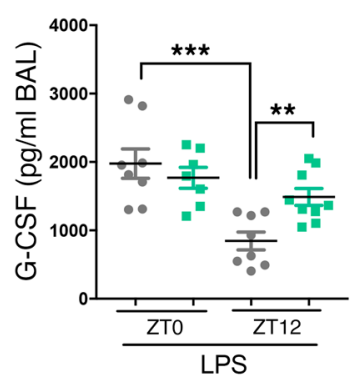


Figure 4. Loss of both REV-ERB $\alpha$ DBD and REV-ERB $\beta$ in the airway epithelium further exaggerates inflammation. (A) Flow analysis of neutrophils in BAL samples after aerosolized LPS $(2 \mathrm{mg} / \mathrm{ml})$ at ZT4 for 20 minutes. Data presented as mean $\pm \mathrm{SEM} ; n=5-8,{ }^{*} P<0.05$ (Student's $t$ test). (B) Cytokine/chemokine protein levels in BAL samples after aerosolized LPS or saline at ZT4 for 20 minutes. Data presented as mean \pm SEM; $n=5-9,{ }^{* *} P<0.01,{ }^{* * *} P<0.001$ (2-way ANOVA, post hoc Bonferroni). (C) qPCR analysis of $C x c / 5$ levels in lung tissues from the same mice as above. Data normalized to saline Rev-Erba fl/fl control group and presented as mean \pm SEM; $n=5-9$, ${ }^{* *} P<0.001$ (2-way ANOVA, post hoc Bonferroni). (D) Neutrophil numbers in BAL samples after aerosolized LPS or saline at ZT4 for 20 minutes. Data presented as mean \pm SEM; $n=5-9,{ }^{* *} P<0.01$ (2-way ANOVA, post hoc Bonferroni). (E) CXCL5 protein levels in the same BAL samples as above. Data presented as mean \pm SEM; $n=5-9$, ${ }^{* *} P<$ 0.001 (2-way ANOVA, post hoc Bonferroni). (F) Neutrophil numbers in BAL samples collected 5 hours after aerosolized LPS challenge at ZTO or ZT12. Data presented as mean $\pm \mathrm{SEM} ; n=7-9,{ }^{*} P<0.05$, ${ }^{* *} P<0.01$ (2-way ANOVA, post hoc Bonferroni). (G) Cytokine/chemokine protein levels in the same BAL samples as above. Data presented as mean $\pm \mathrm{SEM} ; n=7-9,{ }^{*} P$ $<0.05$, ${ }^{* *} P<0.01,{ }^{* *} P<0.001$ (2-way ANOVA, post hoc Bonferroni). (H) qPCR analysis of $C x c / 5$ levels in lung tissues from the same mice as above. Data normalized to saline Rev-Erb $\alpha / \beta^{f / / f l}$ control group and presented as mean \pm SEM; $n=7-9,{ }^{* * *} P<0.0001$ (2-way ANOVA, post hoc Bonferroni).

mice exhibited near-total abolition of targeted Rev-Erb $\alpha$ mRNA, but expressed the truncated, DBD-deleted Rev-Erbo transcript (Supplemental Figure 4B), consistent with previous findings (14). We cultured circadian-synchronized peritoneal macrophages, and the targeted deletion did not impair core circadian oscillations of PER2::Luc bioluminescence (Supplemental Figure 4C).

We did not detect differences between the LysM-Rev-Erb $\alpha$ $\mathrm{DBD}^{\mathrm{m}}$ mice and littermate controls in the inflammatory response to aerosolized LPS at ZT4 (Supplemental Figure 4, D and E). To exclude the possibility that we had missed the time at which a difference was present, a phase shift in the response curve, we compared genotypes at 6-hour intervals, but again saw no difference (Supplemental Figure 4F). Further, we deleted Rev-Erb $\beta$ in myeloid cells, and again saw no phenotype (Supplemental Figure $4 \mathrm{G}$ ). From this, we conclude that REV-ERB $\alpha$, in a non-DBDdependent mechanism, in myeloid-lineage cells participates in conferring time-of-day variation in neutrophilic lung inflammation, but that the increased amplitude of response observed in the global knockouts is not seen. Therefore, we concluded that another cell type is required to explain the overall impact of global REVERB $\alpha$ loss, and next examined the role of the bronchial epithelium.

Targeted deletion of the DBD of Rev-Erba in bronchiolar epithelial cells augments pulmonary inflammatory responses. We targeted bronchial epithelial cells by crossing Rev-Erba $a^{f / f}$ mice with a Ccspicre/+ line, which is selectively expressed in pulmonary bronchoepitheliallineage cells (2). As for the mutation in myeloid cells, deletion of REV-ERB $\alpha$ DBD in bronchial epithelial cells did not impair circadian oscillations of PER2::Luc bioluminescence in the bronchioles (Supplemental Figure $4 \mathrm{H})$. Ccsp-Rev-Erb $\alpha-\mathrm{DBD}^{\mathrm{m}}$ mice exhibited a marked and exaggerated neutrophilic response to LPS compared with Rev-Erbofl/fl littermate controls (Figure 2C). To investigate the mediating pathway, we screened a panel of 23 proinflammatory cytokines/chemokines from BAL fluid by multiplex assay, and here only a single chemokine, CXCL5, was significantly increased in Ccsp-Rev-Erb $\alpha-\mathrm{DBD}^{\mathrm{m}}$ mice, both at basal levels and following
LPS (Figure 2D and Supplemental Table 3). RNA analyses for inflammatory genes in whole lung extracts also revealed marked specificity of response for $\mathrm{Cxcl5}$ (Figure 2E). Therefore, bronchial epithelial deletion of the REV-ERB $\alpha$ DBD offers a similar but milder phenotype compared with the global REV-ERB $\alpha$ deletion. The differences seen in comparing the global loss of REV-ERB $\alpha$ likely reflect contributions from both the epithelium and also the myeloid lineage, and, interestingly, while the DBD of REV-ERB $\alpha$ is required in the epithelium for the effect on the magnitude of inflammation, it is not needed within the myeloid lineage. Aerosolized LPS challenge at ZTO or at ZT12 further identified that the exaggerated inflammatory responses in Ccsp-Rev-Erb $\alpha-\mathrm{DBD}^{\mathrm{m}}$ mice were largely confined to ZTO (Supplemental Figure 4I and Supplemental Table 4).

Dual targeting of Rev-Erb $\alpha$ and Rev-Erb $\beta$ in CCSP-expressing cells abolishes diurnal rhythmicity in the airway epithelium and exaggerates inflammatory responses. Both REV-ERB $\alpha$ and its paralog REVERB $\beta$ are recognized as partially redundant elements driving the core circadian oscillator, with disruption of both genes leading to loss of circadian rhythmicity $(13,23)$, which makes them bona fide core clock genes. To determine the effects of possible functional redundancy between the REV-ERBs, both of which require BMAL1 for expression, mice with conditional deletion of both Rev-Erba DBD and Rev-Erb $\beta$ in bronchiolar epithelial cells (Ccsp-Rev-Erbo$\left.\mathrm{DBD}^{\mathrm{m}} / \operatorname{Rev}-\mathrm{Erb}^{-1-}\right)$ were generated by breeding of Rev-Erbat/fl/ $R e v-E r b \beta^{\text {R/fl }}$ mice with $C c s p^{i c r e /+}$ mice, with all strains bred on a PER2::Luc background. Laser capture of bronchiolar epithelial cells from lungs collected at ZT9 confirmed the efficiency of the gene targeting strategy with marked reduction in the targeted exon transcripts, accompanied by a 12 -fold increase in Bmal1 transcript in the double-mutant mice compared with the littermate controls (Figure 3A). As described in previous studies $(14,24)$, this led to an increase in transcript levels of a DBD-deleted Rev-Erb $\alpha$ transcript, which is translated, but no change in the expression of an exon 4deleted Rev-Erb $\beta$ transcript, which is not translated, in the bronchiolar epithelial cells (Supplemental Figure 5A). As we had seen an induction of LPS response in epithelial-targeted mice, we also tested for epithelial expression of the TLR4 gene, but saw no change in expression even in the double-mutant animals (Figure 3A).

In whole lung, we did not detect transcript changes for either Rev-Erb gene, but there was nonetheless a marked upregulation of Bmal1 mRNA, indicating loss of REV-ERB negative feedback (Figure 3B). Bioluminescent imaging of ectopic lung slices from CcspRev-Erb $\alpha-\mathrm{DBD}^{\mathrm{m}} / \mathrm{Rev}-\mathrm{Erb} \beta^{-1}$ mice showed disrupted circadian oscillations of PER2::Luc in the bronchioles (Figure 3C). This loss of rhythmicity was confined to the bronchioles, as PER2::Luc oscillations were retained elsewhere in the lung parenchyma (Figure 3D).

We next compared inflammatory responses to aerosolized LPS at ZT4 in Ccsp-Rev-Erb $\alpha-\mathrm{DBD}^{\mathrm{m}}$ versus Ccsp-Rev-Erb $\alpha-\mathrm{DBD}^{\mathrm{m}} /$ Rev-Erb $\beta^{--}$mice, with littermate controls $\left(\mathrm{CCSP}^{+/+}\right)$. Doublemutant mice exhibited larger neutrophilic responses to LPS (2-fold), compared with their littermate controls (Figure 4A), than Ccsp-Rev$E r b \alpha-D_{B D} \mathrm{~m}$ mice (1.4-fold; Figure 2A). Only a single chemokine (CXCL5) was differentially expressed in Ccsp-Rev-Erbo-DBD ${ }^{\mathrm{m}}$ mice in response to LPS (Figure 4, B and C, and Supplemental Table 5), consistent with the previous experiments. CXCL5 expression (both protein and transcript) was greatly increased in 

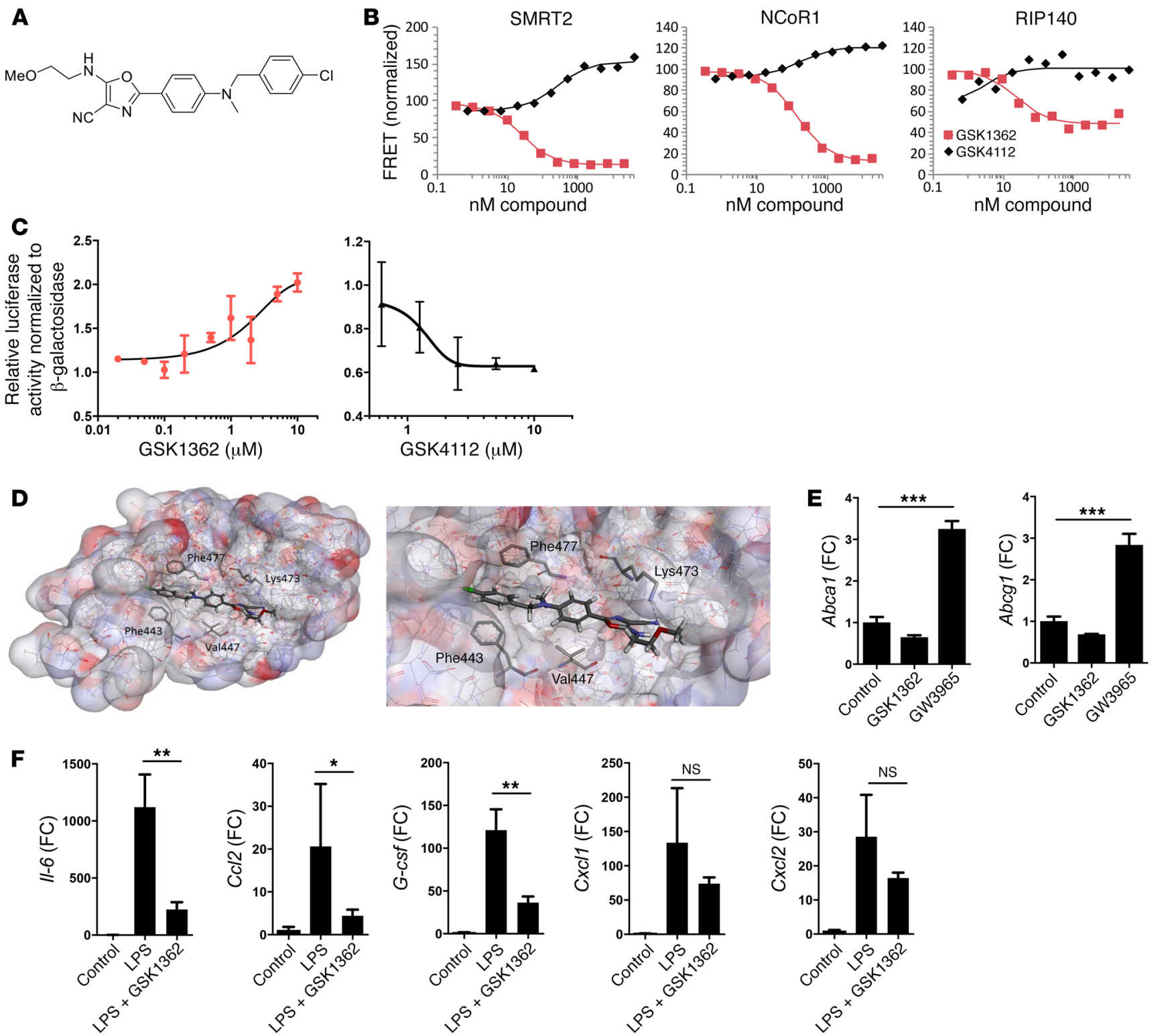

G

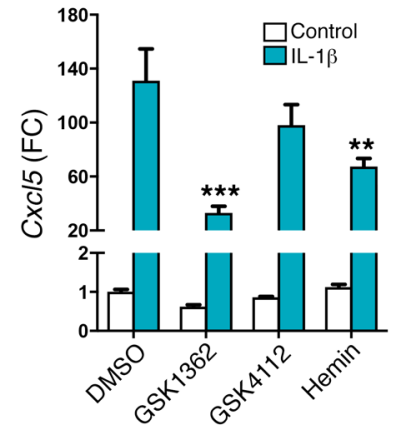

H

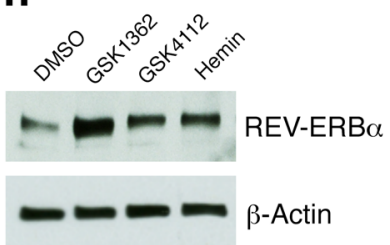

I

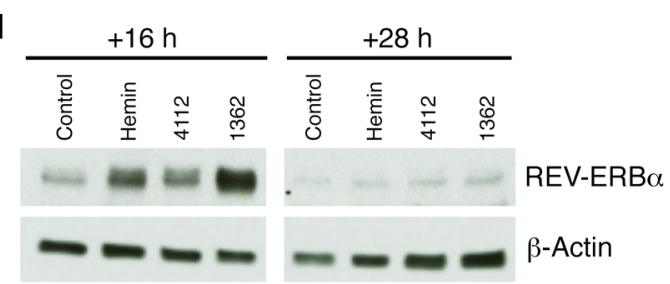


Figure 5. REV-ERB $\alpha$ ligand GSK1362 represses inflammatory genes in macrophages and epithelial cells and stabilizes REV-ERB $\alpha$ protein. (A) Chemical structure of GSK1362. (B) Effect of CSK1362 and GSK4112 on peptide fragment recruitment to REV-ERB $\alpha$. (C) Cotransfection of HEK293 cells with HA-Rev-Erb $\alpha$ and Bmal1-Luc reporter. Cells were treated with GSK1362 or GSK4112 at different concentrations for 24 hours before luciferase assay. Values plotted relatively to $0.1 \%$ DMSO; error bars indicate mean \pm SD. Data representative of $n=3$. (D) Models showing GSK1362 docked in REV-ERB $\alpha$ ligand-binding domain. (E) qPCR analysis of LXR target genes in peritoneal exudate cells treated ex vivo with GSK1362 at $10 \mu \mathrm{M}$ or CW3965, a standard LXR agonist, at $2 \mu \mathrm{M}$ for 4 hours. Data presented as mean $\pm \mathrm{SD} ; n=3$, ${ }^{* *} P<0.001$ (1-way ANOVA, post hoc Bonferroni). (F) qPCR analysis of cytokine mRNA in alveolar macrophages collected at ZT8, seeded into plates and directly treated with GSK1362 at $10 \mu \mathrm{M}$ in the presence or absence of LPS at $100 \mathrm{ng} / \mathrm{ml}$ for 4 hours. Data presented as mean $\pm \mathrm{SD} ; n=3,{ }^{*} P<0.05,{ }^{* *} P<0.01$ (1-way ANOVA, post hoc Bonferroni). (C) qPCR analysis of $C x c / 5$ in LA-4 cells synchronized by serum shock and treated 16 hours later with ligands at $10 \mu \mathrm{M}$, followed 2 hours later by IL-1 $\beta$ at $1 \mathrm{ng} / \mathrm{ml}$ for 2 additional hours. Data normalized to unstimulated control cells and presented as mean $\pm S D$; representative of $n=3,{ }^{* *} P<0.01$, ${ }^{* * *} P<0.001$ (2-way ANOVA, post hoc Bonferroni). (H) REV-ERB $\alpha$ protein in LA- 4 cells synchronized by serum shock and treated 16 hours later with ligands at $10 \mu \mathrm{M}$ for 4 hours. Representative of $n=3$. (I) REV-ERB $\alpha$ protein in NHBE cells synchronized by serum shock and treated 16 or 28 hours later with ligands at $10 \mu \mathrm{M}$ for 4 hours. Representative of $n=3$.

Ccsp-Rev-Erb $\alpha-\mathrm{DBD}^{\mathrm{m}} / \mathrm{Rev}-E r b \beta^{-/}$mice, as was the cellular inflammatory reaction to LPS. In these double-mutant mice, in addition to CXCL5, we also observed augmentation of CXCL1, CXCL2, and G-CSF production (Figure $4 \mathrm{~B}$ ) - the same 4 chemokines that showed altered expression in the global Rev-Erbo-null mice. Further, in Ccsp-Rev-Erb $\alpha-\mathrm{DBD}^{\mathrm{m}} / \mathrm{Rev}-E \mathrm{E} b \beta^{-/-}$mice we also observed significantly increased CXCL5 in unchallenged conditions, equivalent to that seen in control Rev-Erb $\alpha / \beta^{f / f l}$ littermate mice exposed to LPS (Figure 4B). In situ hybridization of lung sections from unchallenged mice demonstrated that enhanced Cxcl5 expression in Ccsp-Rev-Erb $\alpha-\mathrm{DBD}^{\mathrm{m}} / \mathrm{Rev}-E r b \beta^{-/}$mice was confined to the bronchioles (Supplemental Figure 5B), the same structures showing Rev-Erb gene disruption, whereas we did not observe any change in systemic levels of CXCL5 (Supplemental Figure 5C).

The exaggerated inflammatory response observed in the doublemutant animals compared with mice with only mutation of REV$\mathrm{ERB} \alpha \mathrm{DBD}$ could be the consequence of either an additive contribution of REV-ERB $\beta$ or dysregulation of the clock machinery in the bronchioles, the result of disruption to both Rev-Erb genes. To answer that question, we generated mice with conditional deletion of only REV-ERB $\beta$ in bronchiolar epithelial cells (Ccsp-Rev-Erb $\left.\beta^{-/}\right)$ and exposed these animals and their littermate controls (Rev-Erb $\left.\beta^{t / f f}\right)$ to an aerosolized LPS challenge at ZT4. Both groups exhibited similar inflammatory responses (Figure 4, D and E). This suggests that $R e v$-Erb $\alpha$ plays the dominant role in regulation of epithelial immunity in the lung, and furthermore that the more severe inflammatory phenotype observed in the double mutant is a likely consequence of a major disruption of circadian timing in these cells.

Further analysis of the double-mutant mice by time of day revealed exaggerated neutrophilic inflammation at both ZTO and ZT12 (Figure 4F). The differences were most pronounced for CXCL5 for both the secreted chemokine and mRNA expression (Figure 4, G and H, and Supplemental Table 6), but smaller differences were also observed for CXCL1, CXCL2, and G-CSF at ZT12 in comparison with littermate controls (Figure 4G and Supplemental Table 6), indicating the impact of loss of overall clock control of inflammatory responses. These data suggest that REV-ERB $\alpha$ has evolved a specific role to couple rhythmic output from the circadian clock to innate epithelial immunity, while REV-ERB $\beta$ 's contribution emerges only in double knockouts, conditions that also cause loss of core cellular circadian clock oscillations, and therefore brings into play a greater diversity of clock output pathways. Importantly, we did not observe any change in the circulating neutrophil pool in response to airway impairment of both REV-ERBs (Supplemental Figure 5D). This indicates that it is local recruitment of neutrophils into the lung rather than disruption of neutrophil production and release into the circulation that explains the lung inflammatory phenotype.

Development and biological action of a novel oxazole inverse agonist of REV-ERB. The availability of specific ligands permits new insight into nuclear receptor biology. Previous attempts to target the REV-ERBs have identified agonists with variable off-target effects on LXRs (15) and low efficacy. In pursuit of new chemical biology tools, we developed a specific ligand with inverse agonist properties: GSK3201362, hereafter referred to as GSK1362 (Figure 5A and Supplemental Figure 6A). Using an established fluorescence resonance energy transfer (FRET) assay (15), recruitment of comodulator peptide sequences to REV-ERB $\alpha$ protein in the presence of GSK1362 was determined, and compared with the previously reported tertiary amine agonist GSK4112 (Figure 5B). GSK1362 inhibited interaction of the REV-ERB $\alpha$ ligand-binding domain with peptides derived from NCoR1 and SMRT2, two repressive comodulators, characteristic actions of an inverse agonist. The interaction of a peptide derived from RIP140, a comodulator for NF-kB/RelA-dependent cytokine gene expression, with $\mathrm{REV}-\mathrm{ERB} \alpha$ was also repressed in a dose-dependent manner. In contrast, the agonist compound GSK4112 promoted the recruitment of NCoR1 and SMRT2 and did not regulate recruitment of the RIP140 peptide. The activity of GSK1362 was also compared with that of GSK4112 using a Bmal1 reporter assay. GSK1362 concentration-dependently increased transcription, while GSK4112 caused inhibition (Figure 5C), suggesting an inverse agonist effect of the compound acting to relieve BMAL1 repression by endogenous REV-ERB ligands such as heme. To gain additional information on GSK1362 engagement with REV-ERB $\alpha$ protein, we established a cellular thermal shift assay (CETSA), which revealed a change in REV-ERB $\alpha$ protein stability resulting from GSK1362 exposure (Supplemental Figure 6B). CETSA is a simple, robust, and agnostic assay, which reports changes in protein structure induced by ligand binding (25). A model for GSK1362 bound to REV-ERB $\alpha$ was constructed from the crystal structure of REVERB $\alpha$ bound to NCoR ID1 (26) (pdb 3NO0) (Figure 5D). Key REVERB interactions with the ligand are driven by the highly hydrophobic ligand-binding domain of the protein and include the terminal 4-chlorobenzyl group flanked by Phe 433 and Phe477 along with the central aromatic ring interacting with Val447. The $O$-methyl ethanolamine side chain of the oxazole proved crucial for activity, which could interact through a key hydrogen bond with Lys473. Importantly, GSK1362 lacked the LXR activity that was seen in first-generation REV-ERB ligands, as it failed to induce expression of the known LXR target genes Abca1 and Abcg1 (Figure 5E). 
A
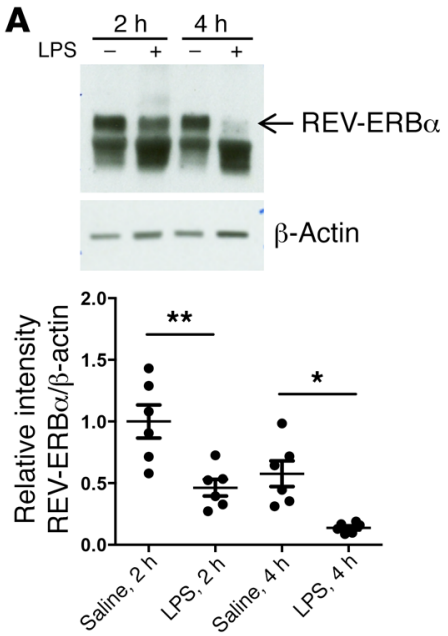

C

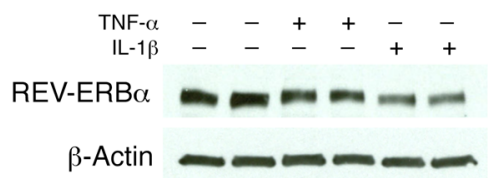

$\mathbf{E}$

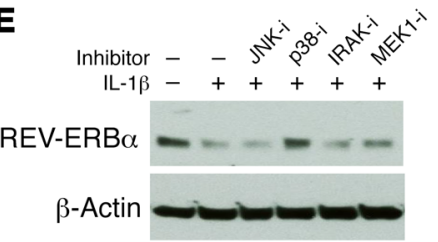

B
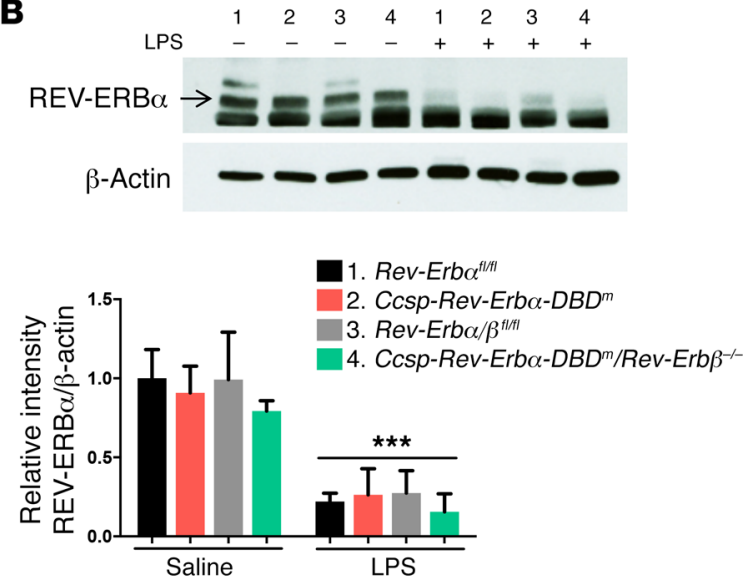

D

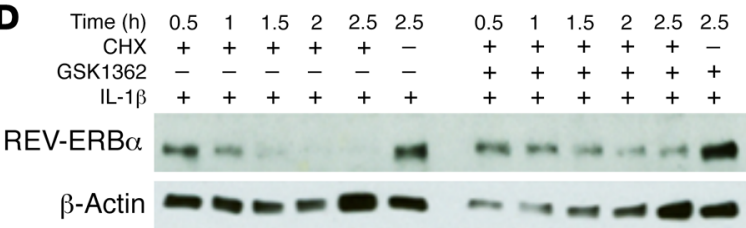

$\mathbf{F}$

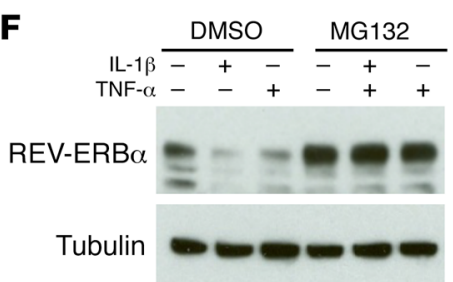

Figure 6. Inflammatory stimuli promote REV-ERB $\alpha$ degradation. (A) Whole-lung REV-ERB $\alpha$ protein in WT mice 2 or 4 hours after aerosolized LPS $(2 \mathrm{mg} / \mathrm{ml})$ or saline solution for 20 minutes at CT8 (see Methods). REV-ERB $\alpha$ densitometry (mean \pm SEM) was normalized to $\beta$-actin and to saline at 2 hours; $n=6,{ }^{*} P<0.05$, ${ }^{* *} P<0.01$ (1-way ANOVA, post hoc Bonferroni). (B) Whole-lung REV-ERB $\alpha$ protein in mice after aerosolized LPS ( $2 \mathrm{mg} /$ $\mathrm{ml}$ ) or saline solution for 20 minutes at ZT4 for 5 hours. REV-ERB $\alpha$ densitometry (mean \pm SD) was normalized to $\beta$-actin and to group $1 ; n=3$, ${ }^{* *} P<0.001$ (2-way ANOVA, post hoc Bonferroni). (C) REV-ERB $\alpha$ protein in NHBE cells synchronized by serum shock and treated 18 hours later with TNF- $\alpha$ or IL- $1 \beta$ at $10 \mathrm{ng} / \mathrm{ml}$ for 1 hour. (D) REV-ERB $\alpha$ protein in NHBE cells synchronized by serum shock and treated 18 hours later with GSK1362 or DMSO at $10 \mu \mathrm{M}$ followed 15 minutes later by cycloheximide (CHX) at $10 \mu \mathrm{M}$ and IL-1 $\beta$ at $1 \mathrm{ng} / \mathrm{ml}$. Cells were lysed at different times as indicated. (E) REV-ERB $\alpha$ protein in SW1353 cells synchronized by serum shock and treated 23 hours later with kinase inhibitors for 30 minutes followed by IL-1 13 at $5 \mathrm{ng} / \mathrm{ml}$ for 1 hour. (F) REV-ERB $\alpha$ protein in SW1353 cells synchronized by serum shock and treated 23 hours later with PBS, TNF- $\alpha$, or IL-1 $1 \beta$ at $5 \mathrm{ng} / \mathrm{ml}$ for 1 hour in the absence and presence of MC132 at $5 \mu \mathrm{M}$. All blots are representative of at least $n=3$.

We previously demonstrated that the REV-ERB agonist GSK4112 inhibited cytokine production from activated macrophages (5). We were therefore surprised to discover that REV-ERB inverse agonist GSK1362 also inhibited LPS induction of several inflammatory cytokines from alveolar macrophages (Figure 5F). In bone marrow-derived macrophages, GSK1362 inhibited Il-6 gene expression in a REV-ERB $\alpha$-dependent manner (Supplemental Figure 7A), but had no effect on $C c l 2, C x c l 1$, or Cxcl2, highlighting the complex effects of REV-ERB ligands. We also used the previously described REV-ERB $\alpha$ antagonist SR8278 (27), and found that this had no effect on $\mathrm{Il}-6$. These studies do not exclude the possibility of further off-target effects of the GSK1362 ligand; therefore, we screened GSK1362 against a panel of 20 nuclear receptors, which did not reveal any significant activity (Supple- mental Table 7). Therefore, we add to evidence that GSK1362 acts through REV-ERB $\alpha$, but GSK1362 cannot be regarded as a chemical probe, as it may have additional targets, which remain to be defined.

Extending our analysis to bronchial epithelial cells, GSK1362 inhibited Cxcl5 transcript induction in mouse LA-4 cells, as did, to a lesser extent, the REV-ERB natural ligand hemin (Figure $5 G$ ), providing an additional line of evidence that Cxcl5 is a REV-ERB target gene in airway epithelial cells. In contrast, the REV-ERB ligands had no major effect on other inflammatory genes (Supplemental Figure 7B). Unfortunately, despite exhaustive efforts and trying multiple antibodies, we were not successful in determining highconfidence ChIP-Seq results for endogenous REV-ERB $\alpha$ in LA-4 cells, which would have helped us to uncover potential recruitment of REV-ERB $\alpha$ to the $C x c l 5$ gene promoter or enhancer regions in airway epithelial cells.

While these studies of REVERB ligand action were under way, we noted a marked increase in REV-ERB $\alpha$ protein abundance with all ligands tested, but, more importantly, with the novel ligand GSK1362 (Figure 5H). This was also seen in human primary bronchial epithelial (NHBE) cells, which have intact circadian REV-ERB $\alpha$ oscillations (Figure 5I and Supplemental Figure 7, $\mathrm{C}$ and D). However, in NHBE cells, the 2 REV-ERB ligands GSK1362 and GSK4112 had complex and divergent effects on multiple proinflammatory cytokines upon IL-1 $\beta$ stimulation (Supplemental Figure $7 \mathrm{E}$ ), again emphasizing the critical role of cell type-specific factors in determining the action of REV-ERBs, the limitations of REV-ERBs as drug targets for inflammatory diseases, and important differences in response between cell types.

Rapid degradation of REV-ERBa protein is mediated by inflammatory stimuli, and reversed by the inverse agonist GSK1362. While studies have highlighted the importance of the clock in modulation of inflammation, there is evidence that this connection is bidirectional and the inflammatory response itself can affect molecular clock pathways $(28,29)$. As REV-ERB ligands both repressed inflammatory responses and increased REV-ERB $\alpha$ protein concen- 


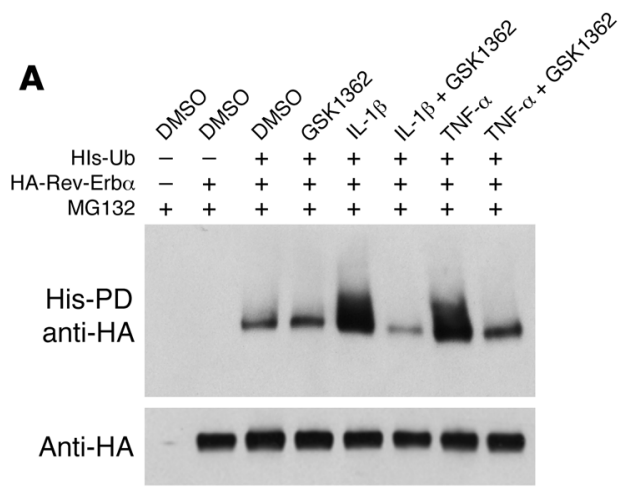

B

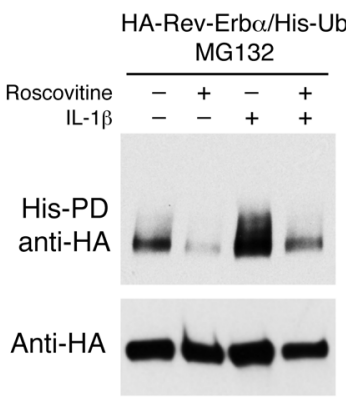

D

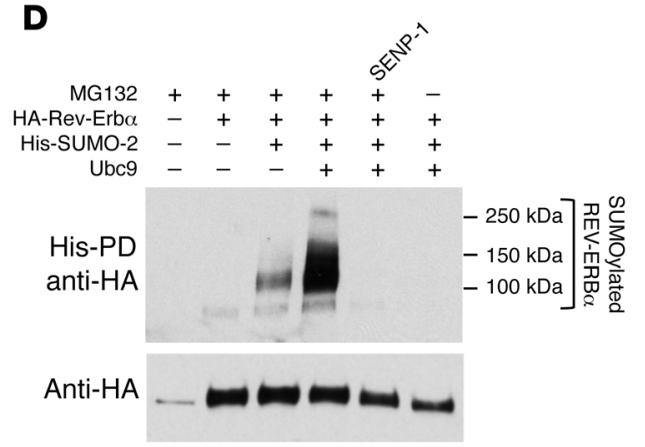

E

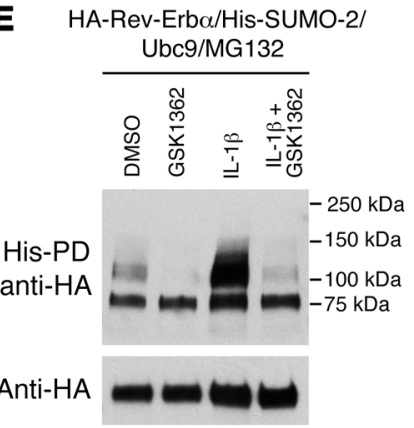

C

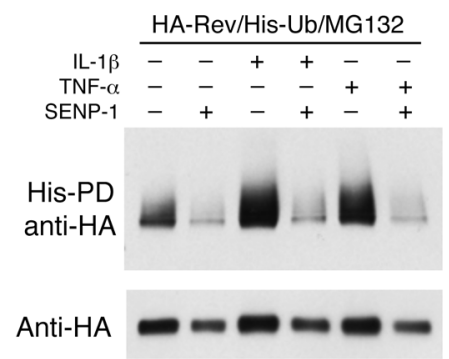

$\mathbf{F}$

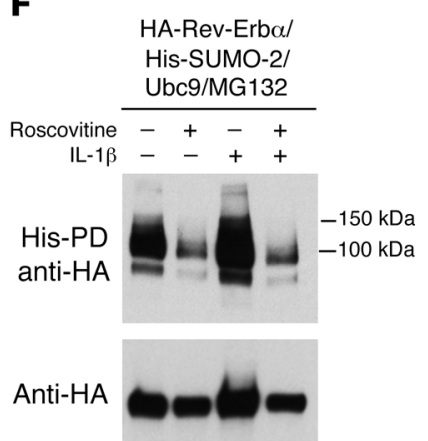

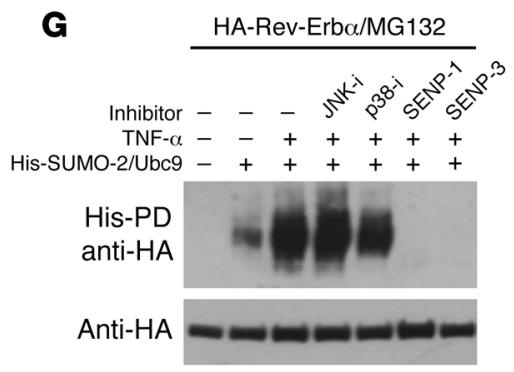

H

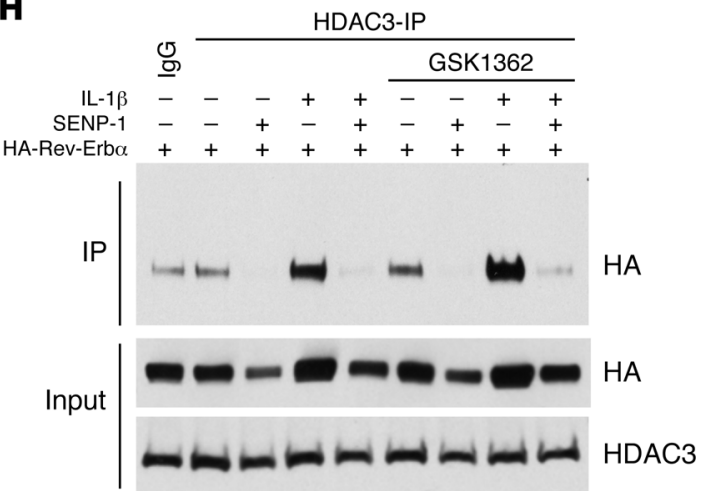

Figure 7. Requirement of posttranslational modifications for REV-ERB $\alpha$ degradation. (A-C) Ubiquitinated REV-ERB $\alpha$ protein in HEK293T cells transfected with HA-Rev-Erb $\alpha$, His-Ub, and SENP-1 plasmids and treated with GSK1362 at $10 \mu \mathrm{M}$, roscovitine at $25 \mu \mathrm{M}$, and TNF- $\alpha$ or IL-1 $\beta$ at $5 \mathrm{ng} / \mathrm{ml}$ for 4 hours in the presence of MG132 at $5 \mu \mathrm{M}$. (D-C) SUMO-2 ligation to REV-ERB $\alpha$ protein in HEK293T cells transfected with HA-Rev-Erb $\alpha$, His-SUMO2, Ubc9, and SENP-1 plasmids and treated with GSK1362 at $10 \mu \mathrm{M}$ and kinase inhibitors and IL-1 $1 \beta$ or TNF- $\alpha$ at $5 \mathrm{ng} / \mathrm{ml}$ for 4 hours in the presence of MG132 at $5 \mu$ M. (H) Coimmunoprecipitation of HDAC3 and REV-ERB $\alpha$ protein in HEK293T cells transfected with HA-Rev-Erb $\alpha$ and SENP-1 plasmids and treated with GSK1362 at $10 \mu \mathrm{M}$ and IL-1 $\beta$ at $5 \mathrm{ng} / \mathrm{ml}$ for 4 hours. All blots are representative of at least $n=3$.

tration, the effect of inflammation on REV-ERB $\alpha$ protein was determined. Previous reports support inhibition of REV-ERB $\alpha$ transcription by inflammation $(11,30)$, but here we observed very rapid loss of REV-ERB $\alpha$ protein in inflamed lung tissue (Figure 6A). We also found that the REV-ERB $\alpha$ protein lacking its DBD also showed such degradation, again in lung tissue (Figure 6B). This was mimicked by inflammatory cytokine action in vitro (Figure $6 \mathrm{C}$ ), and was opposed by GSK1362 (Figure 6D). Therefore, GSK1362 stabilizes REV-ERB $\alpha$ protein, identifying an important site of inflammatory/ circadian crosstalk, an effect mediated, in part, by inflammatory activation of p38 MAP kinase (Figure 6E). REV-ERB $\alpha$ protein levels were greatly increased when cells were treated with the protea- some inhibitor MG132 (Figure 6F), identifying the $26 \mathrm{~S}$ proteasome as promoting rapid REV-ERB $\alpha$ protein degradation.

Posttranslational mechanisms are required for REV-ERB $\alpha$ degradation, and are blocked by the inverse agonist GSK1362. Posttranslational modifications such as phosphorylation, SUMOylation, and ubiquitination constitute an important regulatory system in nuclear receptor function. The rapid, proteasomal degradation of REV-ERB $\alpha$ induced by inflammatory cytokine action suggests a ubiquitination step. Indeed, REV-ERB $\alpha$ was rapidly ubiquitinated in response to either IL-1 $\beta$ or TNF- $\alpha$ action, an effect blocked by GSK1362 (Figure 7A). Interestingly, ubiquitination of REV-ERB $\alpha$ upon IL-1 $1 \beta$ treatment was also prevented by the CDK inhibitor 
roscovitine (Figure 7B), suggesting that the CDK1/FBXW7 pathway targeting REV-ERB $\alpha$, described in previous studies (31), is activated by inflammatory cytokines. In addition to CDK inhibition impairing ubiquitination, the SUMO protease SENP-1 was also highly effective (Figure 7C), indicating that SUMOylation is a requirement for REV-ERB $\alpha$ ubiquitination, and prompting further investigation into REV-ERB $\alpha$ SUMOylation, which revealed Ubc9-dependent SUMO-2 ligation (Figure 7D). IL-1 $\beta$ promoted SUMO-2 ligation to REV-ERB $\alpha$, whereas ligand GSK1362 blocked it (Figure 7E), and again inhibition of CDKs with roscovitine blocked SUMOylation (Figure 7F), supporting a pathway of phosphorylation followed by SUMO-dependent ubiquitination. As we had identified p38 inhibition to block inflammatory cytokine-driven REV-ERB $\alpha$ degradation, we looked for a p38 role in SUMOylation also, and indeed showed that p38 inhibition, but not JNK inhibition, reduced REV-ERB $\alpha$ SUMOylation (Figure 7G).

Inflammatory signaling also drove a SUMOylation-dependent recruitment of HDAC3 to REV-ERB $\alpha$, an effect that was not disturbed by GSK1362 ligand binding (Figure 7H). The failure of ligand binding to regulate HDAC3 recruitment suggests that the principle mechanism of ligand action to suppress inflammatory cytokine expression is by stabilizing the REV-ERB $\alpha$ protein under conditions favoring rapid degradation. We attempted to localize the modified lysine in REV-ERB $\alpha$ using a mutagenesis approach, but were not able to identify a single, dominant lysine. We consider it likely that the REV-ERB $\alpha$ protein is multiply modified, with a complex code including phosphorylation, SUMOylation, and SUMO-dependent ubiquitination.

Analysis of REV-ERB $\beta$ modification, in contrast, revealed only a very minor increase in ubiquitination in response to IL-1 $\beta$ (Supplemental Figure 8A), and we could not detect SUMOylation under any circumstance (Supplemental Figure 8B). This again fits with the divergence of function, with REV-ERB $\alpha$ playing the dominant role in this signaling circuit.

\section{Discussion}

The circadian clock plays a vital role in coordinating many physiological programs across tissues in anticipation of predictable changes in the environment, principally day-night. The role of such biological timing mechanisms in inflammation and immunity is being defined now, with the most prominent phenotypes centering on macrophages and innate immunity. We have previously defined the role of BMAL1, the only essential core clock component for cell-autonomous oscillations, in pulmonary inflammation and immunity. Moreover, we defined a surprising dominant role for timing circuits in the airway epithelium, rather than in the myelomonocytic lineage, by disrupting BMAL1 in these cells. However, the role of BMAL1 versus loss of timing, and the downstream pathways regulating inflammatory signaling, remained key unanswered questions. We now identify a highly specific circuit requiring the nuclear receptor and tractable drug target REV-ERB $\alpha$ that mediates the BMAL1 effect within bronchial epithelial cells to regulate pulmonary inflammation.

Global loss of REV-ERB $\alpha$ resulted in exaggerated pulmonary, innate immune responses with loss of the normal evening nadir in inflammatory response to LPS. Analysis of the myeloid cell population revealed that complete loss of REV-ERB $\alpha$ had a modest effect on pulmonary neutrophilic inflammation, with attenuation of the time-of-day variation. In these studies, we compared analysis of BAL with lung digest, as a means to recover immune cells from inflamed lung, and confirmed that the digest offers a more precise assay. Indeed, we only observed the loss of time-of-day variation in lung digest analysis.

Mutation of the REV-ERB $\alpha$ DBD in myeloid cells had no impact on pulmonary inflammatory responses in our in vivo model, which was unexpected, both because isolated alveolar macrophages lacking REV-ERB $\alpha$ show exaggerated LPS responses ex vivo, and because complete loss of REV-ERB $\alpha$ did have a phenotype. Therefore, we conclude that the REV-ERB $\alpha$ effect in myeloid cells is exerted through a non-DBD-dependent mechanism, akin to that documented in the liver, where DBD-independent control of lipid metabolism was seen to dissociate from the clock-regulating functions of REV-ERB $\alpha$, which require binding to consensus REV-ERB cis elements (14). Deletion of REV-ERB $\beta$ in the myeloid lineage did not result in a lung inflammatory phenotype. In contrast, mutation of REV-ERB $\alpha$ DBD in bronchiolar epithelial cells resulted in increased neutrophilia and CXCL5 levels. This suggests a prominent conditioning role for the airway epithelial cells in vivo and indicates that REV-ERB $\alpha$ in bronchiolar epithelial cells possesses an antiinflammatory function, which is operated by its ability to bind DNA. Mice with complete loss of REV-ERB $\alpha$ in bronchiolar epithelial cells are not yet available but would be particularly useful to determine a potential non-DNA-binding effect of REV-ERB $\alpha$ in the airway epithelium upon gating of the inflammatory responses, similar to the effect observed in REV-ERB $\alpha$-null myeloid cells.

As both REV-ERB $\alpha$ and REV-ERB $\beta$ show major physiological redundancy, the question of REV-ERB $\beta$ function required analysis. Using a double-mutant mouse model, we found a significant compensatory role for REV-ERB $\beta$, with greatly exaggerated LPS responses, involving more chemokines, and also, intriguingly, leading to augmented CXCL5 production under basal conditions. However, we discovered that the REV-ERB $\alpha$ paralog is dominant over the REV-ERB $\beta$, which could be deleted in the bronchial epithelium without a resulting inflammatory phenotype.

There has been great interest in understanding how the circadian clock affects immunity $(7,8,32)$. BMAL1 deletion in the pulmonary epithelium blocks circadian control of inflammation, and augments the inflammatory response. BMAL1 plays many roles in development beyond its circadian function, and indeed postnatal BMAL1 loss results in a far milder phenotype than constitutive deletion (33). Some studies have revealed a direct role for BMAL1 acting to regulate NF-kB function either through enzymatic modification (9) or through regulation of microRNA (10). In our current study, we show that functional disruption/deletion of the 2 REVERB paralogs is proinflammatory, despite a massive increase in BMAL1 expression, with the same CXCL neutrophil chemokines and G-CSF emerging as key inflammatory mediators. These new data demonstrate a functionally important pathway from the core clock through REV-ERB $\alpha$ to regulate pulmonary inflammation. However, further studies will be required to establish with high confidence REV-ERB $\alpha$ cistrome in airway epithelial cells and uncover relevant transcriptional mechanisms.

Functional analysis of nuclear receptors can be greatly accelerated by use of regulating ligands, and previous attempts to tar- 
get REV-ERB have been promising. However, early-generation ligands had significant LXR liability, prompting us to develop a new compound with no LXR action, but with a unique pattern of comodulator peptide recruitment. In vitro, GSK1362 promoted disruption of repressor peptide interaction and so is an inverse agonist, an activity supported by divergent effects on Bmal1 promoter regulation in comparison with GSK4112, an agonist. GSK1362 was tested on both isolated macrophages and bronchial epithelial cells, with divergent and complex results, some of which may result from off-target actions. We screened GSK1362 against a 20-nuclear receptor panel, and found negligible activity. We also used a CETSA assay to gain additional information on engagement with REV-ERB $\alpha$. Our sufficiency studies were necessarily limited in scope, but provided evidence that REV-ERB $\alpha$ was required for regulation of $\mathrm{Il}-6$, a well-characterized REV-ERB $\alpha$ target gene in macrophages. Therefore, GSK1362 is a useful chemical tool, capable of binding to REV-ERB $\alpha$ and regulating its function. However, we do not claim GSK1362 has all the criteria for use as a chemical probe for REV-ERB $\alpha$.

While investigating the mechanism of GSK1362 action we discovered a surprising ligand-dependent increase in REV-ERB $\alpha$ protein, suggesting that the observed chemokine repression seen with the ligand may in part be due to increased abundance of the repressor REV-ERB $\alpha$ protein.

We discovered that inflammation promotes degradation of $\mathrm{REV}-\mathrm{ERB} \alpha$ protein, through SUMO-dependent ubiquitination of the protein and involvement of CDKs and p38 MAP kinase. Strikingly, the inflammation-driven modification of REV-ERB $\alpha$ was efficiently blocked by GSK1362, which therefore resulted in an increase in REV-ERB $\alpha$ protein, and more efficient inflammatory mediator gene repression. Unfortunately, GSK1362 cannot be used in vivo because of adverse predicted PK characteristics. GSK1362 is therefore a useful chemical biology tool for interrogating REV-ERB $\alpha$ function, but whether it will be possible to make a useful human therapeutic targeting REV-ERB remains an open question. In vivo effects of other REV-ERB $\alpha$ ligands have been reported $(34,35)$, but these recent studies did not compare the effects in REV-ERB $\alpha$-null mice, making it impossible to attribute the phenotypes observed to a specific action on REV-ERB $\alpha$.

Taken together, our data illuminate a new circuit lying between the core circadian clock and inflammation, with both major networks converging on REV-ERB $\alpha$ protein, an obligate repressor of aspects of the pulmonary inflammatory response. Identification of $\mathrm{REV}-\mathrm{ERB} \alpha$ degradation as a robust early response to inflammation supports a homeostatic role in determining the inflammatory set point, limiting inflammatory activity under resting, nonstress conditions, as evidenced by the high basal production of chemokines in the bronchial epithelium of double-mutant mice (Supplemental Figure 9). REV-ERB $\alpha$ has been proposed as a tractable drug target for metabolic disease, and also possibly for inflammatory disease. The highly variable target gene responses seen with the new ligand suggest that any such therapeutic development would require careful consideration, not least as the pattern of response differs between cell types. However, targeting of the bronchial epithelium is tractable via an inhalation approach, although the failure of REV-ERB $\alpha$ disruption to impact on allergic inflammation, the major human disease burden, would temper enthusiasm. We propose that REV-ERB $\alpha$ degradation by inflammatory signaling offers a new pathway to explain circadian disruption in chronic inflammatory disease, and the REV-ERB $\alpha$ stabilization seen with ligand binding offers a new mechanism for REV-ERB $\alpha$ functional regulation. In addition, the emerging role of REV-ERB $\alpha$ as a cell type-specific mediator of timing information and control of inflammatory response gives new insights into an ancient hostdefense control system.

\section{Methods}

Animals. Nr1d1 ${ }^{\text {tmIschb }}$ mice with global Rev-Erb $\alpha$ knockout (subsequently known as Rev-Erb $\alpha^{-/}$) were provided by Ueli Schibler (University of Geneva, Geneva, Switzerland) (16). These animals were bred as a heterozygous colony, and offspring genotyped to identify knockout and WT animals. Conditional club cell or myeloid cell Rev-Erb $\alpha$-DBD mutant mice (Ccsp-Rev-Erb $\alpha-\mathrm{DBD}^{\mathrm{m}}$ or LysM-Rev-Erb $\left.\alpha-\mathrm{DBD}^{\mathrm{m}}\right)$ were generated by breeding of Rev-Erbo ${ }^{f / f l}$ mice (Nr1d1 ${ }^{\text {tmIIcs; }}$ Institut Clinique de la Souris, Illkirch, France) with Ccsp-iCre (36) or LysM-Cre mice. Similarly, conditional club cell Rev-Erb $\alpha$-DBD mutant and Rev-Erb $\beta$ knockout mice (Ccsp-Rev-Erb $\left.\alpha-\mathrm{DBD}^{\mathrm{m}} / \operatorname{Rev}-E r b \beta^{-/}\right)$were generated by breeding of Rev-Erb $\beta^{f l f l}$ mice (Nr1d2 $2^{\text {tml.1Rev }}$; Institut Clinique de la Souris) with Ccsp-Rev-Erb $\alpha-\mathrm{DBD}^{\mathrm{m}}$ mice. Tissue expression of iCre (CCSP) or Cre (LysM) was assessed by reverse transcriptase PCR as described elsewhere $(2,5)$. All mouse lines were subsequently crossed onto a PER2::Luc background (37). All animals were housed in a 12-hour light/12-hour dark schedule with food and water available ad libitum. In all studies, both male and female mice were used (age 6-12 weeks).

Aerosolized LPS challenge. In vivo LPS challenge was undertaken as described previously (2). In brief, animals were exposed to an aerosol of LPS (E. coli 0127:B8; Sigma-Aldrich) at doses ranging from 0.05 $\mathrm{mg} / \mathrm{ml}$ to $2 \mathrm{mg} / \mathrm{ml}$ for 20 minutes. Animals were sacrificed 5 hours later. Bronchoalveolar lavage (BAL) was performed by instillation and removal of $1 \mathrm{ml}$ aliquot of BAL fluid (10 mM EDTA in PBS with $0.1 \%$ BSA) administered via a tracheal cannula. Lung tissues were collected and snap-frozen on dry ice or were filled with paraformaldehyde and removed for histological analysis. Challenges at ZTO and ZT4 were performed under normal light, whereas exposures at ZT12 were performed in constant darkness, using infrared goggles to view animals. Circadian time (CT) refers to time points under continuous darkness, with CTO the start of the biological day, and CT12 the start of the biological night, and activity period in mice.

Smoke exposure. Cigarette smoke exposure was performed as previously described (38). Briefly, mice received a daily 2-hour noseonly exposure to $4 \%$ cigarette smoke from $3 \mathrm{R} 4 \mathrm{~F}$ cigarettes (College of Agriculture, Reference Cigarette Program, University of Kentucky, Lexington, Kentucky, USA). During exposure to smoke or air only (sham controls), mice were maintained in restraining tubes containing stainless steel nose cone inserts. BAL samples and lung tissues were collected as previously described.

Bone marrow transplant study. Recipient mice (B6 Cd45.1, Pep Boy; The Jackson Laboratory) were placed on Baytril antibiotic (SigmaAldrich) for 2 weeks before and 4 weeks after radiation exposure. Donor mice ( $\mathrm{Rev}$-Erb $\mathrm{K}^{-/}$or littermate controls) were culled by cervical dislocation, and femurs/tibiae were removed. Bone marrow was flushed out using Iscove's Modified Dulbecco's Media (supplemented with $2 \%$ FCS) and suspended in red blood cell lysis buffer (Roche). The remaining cells were depleted of T cells using a CD90.1 Positive 
Selection Kit (EasySep). Remaining donor cells were counted and resuspended to $2 \times 10^{7}$ such that each mouse received a tail vein injection of $4 \times 10^{6}$ cells in $200 \mu$ l. Recipient mice were then given 2 doses of radiation of 5.5 Gy separated by 2 hours. Recipient mice then had $200 \mu \mathrm{l}$ of donor cells injected into the tail vein. Mice were weighed daily after injections and left for 3 months to allow for full immune reconstitution prior to aerosolized LPS exposure.

BAL cytokine analysis, cell counts, and flow cytometry. BAL samples were centrifuged and the supernatant utilized for cytokine and chemokine analysis using the Magnetic Luminex Assay (R\&D Systems). The cell pellets were resuspended in $200 \mu \mathrm{l}$ PBA (PBS with 1\% BSA and $0.1 \%$ sodium azide) for subsequent total cell count and analysis by flow cytometry. In brief, $19 \mu \mathrm{l}$ resuspended cells were mixed with $1 \mu \mathrm{l}$ of fluorescent dye solution 18 (ChemoMetec), and total cell number was assessed using NucleoCounter NC-250 (ChemoMetec). Frequency of neutrophils and macrophages was assessed by flow cytometry using anti-Ly-6G (Gr-1)-Alexa Fluor 488 and anti-CD11c-APC antibodies to detect neutrophils and alveolar macrophages, respectively (2). For flow cytometry analyses on lung digests, the left lobe of the lung was placed in $1 \mathrm{ml}$ RPMI (Sigma-Aldrich) containing Liberase (Sigma-Aldrich) and DNase I (Promega) and chopped up with scissors. The homogenate was placed at $37^{\circ} \mathrm{C}$ for 30 minutes on an orbital shaker. Digestion was stopped with the addition of $1 \mathrm{ml}$ RPMI containing $10 \mathrm{mM}$ EDTA (Sigma-Aldrich). Homogenate was passed through a $70-\mu \mathrm{m}$ sieve (Corning) and cells pelleted at $300 \mathrm{~g}$ for 5 minutes at $4^{\circ} \mathrm{C}$. Pellets were resuspended in red blood cell lysis buffer. Remaining cells were pelleted, counted, and plated at $1 \times 10^{6}$ cells for flow cytometry.

Bronchiolar epithelial cell laser capture. The trachea was cannulated postmortem and lungs inflated with $1 \mathrm{ml}$ PBS/OCT mixture (1:1). The trachea was tied off, and the lungs and heart were removed en bloc and snap-frozen. Ten-micrometer sections were cut on the cryostat, and 3-4 sections were placed per polyethylene naphthalate (PEN) membrane slide (3 slides per animal). Before laser dissection, specimen slides were taken out of dry ice and placed into alcoholic solutions (100\% ethanol for 1 minute, $75 \%$ ethanol 3 dips, 50\% ethanol 3 dips, $95 \%$ ethanol 30 seconds, $100 \%$ ethanol 30 seconds, $100 \%$ ethanol 2 minutes). A Leica LMD 6500 laser-capture microdissection machine was used to cut regions around the bronchiolar airways. For tissue collection, 0.5-ml thin-wall PCR tubes were used in the tissue collector, with $30 \mu$ l lysis buffer (TRK buffer supplemented with $\beta$-mercaptoethanol, MicroElute Total RNA kit; Omega Bio-tek). Samples were then stored at $-80^{\circ} \mathrm{C}$ prior to RNA extraction using MicroElute Total RNA kit, following the manufacturer's instructions.

Lung histology. Paraformaldehyde-fixed lung tissue was processed and embedded, and $5-\mu \mathrm{m}$ sections were mounted onto slides. Sections were used for routine $\mathrm{H} \& \mathrm{E}$ staining. Immunohistochemical staining was carried out using antibody raised against NIMP/R14 (to detect neutrophils; Abcam Ab2557); sections were counterstained with hematoxylin. Slides were viewed using a Leica DM2000 microscope and images captured using a Leica IC90E digital camera and software.

Ectopic lung slices. Precision-cut ectopic lung slices $(275 \mu \mathrm{m})$ were prepared as previously described (39). After washes to remove residual agarose, slices were placed onto cell culture inserts (Millicell) within $35-\mathrm{mm}$ dishes containing $1 \mathrm{ml}$ recording medium and sealed with a coverslip. Dishes were then transferred to a $37^{\circ} \mathrm{C}$ incubator housing the photomultiplier tubes (PMTs; H6240 MOD1; Hamamatsu Photonics) or placed under a self-contained Olympus LV200 luminescence microsco- py system fitted with a cooled Hamamatsu C9100-13 EM-CCD camera (Olympus) as previously described (40). Individual regions of interest were delineated using ImageJ software (version 1.41o; $\mathrm{NIH}$ ).

Peptide recruitment profiles. These experiments were undertaken as previously described (15).

Bmal1 luciferase assay. HEK293 cells were transfected with HA-tagged Rev-Erb $\alpha$, a luciferase reporter driven by the Bmal1 promoter, and a $\beta$-galactosidase reporter using polyethylenimine (PEI) (3:1 vol/wt ratio) (41) and left overnight. Cells were treated with GSK1362 at different concentrations or with 0.1\% DMSO for 24 hours followed by the luciferase assay using the Dual-Light Luciferase and $\beta$-Galactosidase Reporter Gene Assay System (Thermo Fisher Scientific) according to the manufacturer's instructions. Luciferase activity was normalized to $\beta$-galactosidase reading for each sample.

His purification. HEK293 cells were transfected with $0.5 \mu \mathrm{g}$ HA-tagged Rev-Erb $\alpha, 0.75 \mu \mathrm{g}$ His-ubiquitin, $0.75 \mu \mathrm{g}$ His-SUMO-2, 0.5 $\mu \mathrm{g}$ SENP-1, and $0.25 \mu \mathrm{g}$ Ubc9 using PEI (3:1 vol/wt ratio) (41) and left overnight. Cells were treated with $5 \mu \mathrm{M}$ MG132 (Sigma-Aldrich), 10 $\mu \mathrm{M}$ GSK1362 ligand, IL1- $\beta$, or TNF- $\alpha$ as indicated. Cells were lysed in either RIPA buffer (50 mM Tris-Cl pH 7.4, 1\% NP-40, 0.25\% sodium deoxycholate, $150 \mathrm{mM} \mathrm{NaCl}, 1 \mathrm{mM}$ EDTA) supplemented with protease and phosphatase inhibitors for input samples or guanidinium $/ \mathrm{HCl}$ buffer I (6 M guanidinium/ $\mathrm{HCl}, 0.1 \mathrm{M} \mathrm{Na}_{2} \mathrm{HPO}_{4} / \mathrm{NaH}_{2} \mathrm{PO}_{4}, 0.01 \mathrm{M}$ Tris, pH8) for His-purified samples. Lysates for His purification were first sonicated using EpiShear Probe Sonicator and cell debris cleared by centrifugation. Supernatants were added to Ni-NTA Agarose beads (Qiagen), previously washed with buffer I and partially bound with BSA at $50 \mathrm{ng} / \mu \mathrm{l}$ (Sigma-Aldrich). Imidazole $(5 \mathrm{mM})$ and $\beta$-mercaptoethanol $(10 \mathrm{mM})$ were also added, and samples were incubated by rotation at room temperature for 2-3 hours. Beads were washed twice with buffer I supplemented with $5 \mathrm{mM}$ imidazole (rotation at room temperature for 20 minutes followed by centrifugation at $400 \mathrm{~g}$ for 2 minutes to remove supernatant). Beads were then washed with urea buffer II ( $8 \mathrm{M}$ urea, 0.1 $\mathrm{M} \mathrm{Na}_{2} \mathrm{HPO}_{4} / \mathrm{NaH}_{2} \mathrm{PO}_{4}, 0.01 \mathrm{M}$ Tris, $\mathrm{pH}$ 6.3) containing $5 \mathrm{mM}$ imidazole (rotating for 10 minutes), followed by another wash with buffer II containing $5 \mathrm{mM}$ imidazole and 0.2\% Triton X-100 (Sigma-Aldrich). Beads were finally washed with PBS, and after centrifugation at $100 \mathrm{~g}$, supernatants were discarded. Beads were mixed with SDS loading dye containing $10 \% \beta$-mercaptoethanol and boiled 10 minutes at $70^{\circ} \mathrm{C}$. Samples were stored at $-80^{\circ} \mathrm{C}$ until run for Western blotting.

Immunoprecipitation. HEK293 cells were transfected with $1 \mu \mathrm{g}$ HA-tagged REV-ERB $\alpha$ and/or $1 \mu \mathrm{g}$ SENP-1 using PEI (3:1 vol/wt ratio) and left overnight. Cells were treated with $10 \mu \mathrm{M}$ GSK1362 ligand and/or $5 \mathrm{ng} / \mathrm{ml} \mathrm{IL-1 \beta}$ for 4 hours. Cells were lysed in either RIPA buffer supplemented with protease and phosphatase inhibitors for input samples or IP lysis buffer (150 mM NaCl, $20 \mathrm{mM}$ Tris- $\mathrm{HCl}, 10 \%$ glycerol, 1\% Triton X-100, 1 mM PMSF, 10 mM $N$-ethylmaleimide (NEM), PhosSTOP (MilliporeSigma), complete EDTA-free protease inhibitor cocktail) on ice and cell debris cleared by centrifugation. One microgram of anti-HDAC3 antibody (Santa Cruz Biotechnology H-99) or $1 \mu \mathrm{g}$ of rabbit $\operatorname{IgG}$ was incubated with protein lysates for 1 hour on a rotating wheel at $4^{\circ} \mathrm{C}$. Antibody complexes were captured by addition of beads (MagReSyn Protein A; ReSyn Biosciences) for 45 minutes at $4^{\circ} \mathrm{C}$. Beads were washed 3 times with IP lysis buffer, and then boiled for 10 minutes in SDS loading dye containing 10\% $\beta$-mercaptoethanol. Beads were cleared using magnetic separator, and supernatants were ready for electrophoresis. 
Generation of REV-ERB a monoclonal antibody. The GSK6F05-2 antibody (Biocat 137359) was generated by conventional 3-month immunization of SJL mice, with His-tagged Rev-ErbA $\alpha$ ligand-binding domain (aa 281-614), purified from E. coli. Protein-specific monoclonal antibodies were identified by ELISA and Western blot screening of single-cell-derived clones.

CETSA assay. HEK293 cells were transfected with HaloTagRev-Erb $\alpha$ (as described above, $10 \mu \mathrm{g}$ plasmid per 10-cm dish). Cells were treated with GSK1362 or DMSO (0.1\% vol/vol) for 1 hour before the media was replaced with $1 \mathrm{ml}$ PBS (containing protease inhibitor cocktail). One-hundred-microliter aliquots of cells (prepared without further washing in PBS) were heated (from $40^{\circ} \mathrm{C}$ to $58^{\circ} \mathrm{C}$ using a thermocycler) for 3 minutes and then incubated at room temperature for a further 3 minutes. Following 2 freeze-thaw cycles (using dry ice, and a thermocycler at $25^{\circ} \mathrm{C}$ ), 2 microliters of $10 \%$ (vol/vol) Triton X-100 was added and the lysate vortexed. Cell lysates were centrifuged at $20,000 \mathrm{~g}$ for 20 minutes $\left(4^{\circ} \mathrm{C}\right)$ to pellet insoluble cell debris. Supernatants were incubated with Halo-ligand (Alexa Fluor 660; Promega) for 15 minutes at room temperature before $4 \times$ lithium dodecyl sulfate (LDS) loading buffer and $100 \mathrm{mM}$ DTT were added. After electrophoresis, gels were visualized using an Odyssey CLx infrared imaging system. Western blotting was carried out with PVDF (Immobilon-FL Membrane; Merck Millipore) and an anti-lamin B1 (1:1,000; Proteintech) antibody. Blots were visualized with an anti-rabbit CF800 secondary antibody (1:20,000; Biotium) and an Odyssey CLx imager.

Statistics. Values are expressed as mean \pm SEM or mean \pm SD as stated. Data were analyzed using GraphPad Prism 6.0. Parametric statistical analyses were applied when data showed normal distribution; otherwise nonparametric tests were used. The statistical analysis was conducted at $95 \%$ confidence level, with a $P$ value less than 0.05 being considered statistically significant $\left({ }^{* *} P \leq 0.001,{ }^{* *} P \leq 0.01\right.$, $\left.{ }^{*} P \leq 0.05\right)$. PMT recordings were normalized to a rolling average before plotting as a function of time; period analysis was carried out using RAP software 50, and Cosinor analysis was carried out using Cosinor.exe version 2.3 (http://www.circadian.org/softwar.html). All animals were randomized to treatment and control groups, and investigators were blinded to genotype and treatment.

Study approval. All animal studies were ethically reviewed and carried out in accordance with standards defined by the Animals (Scientific Procedures) Act, United Kingdom, 1986 or European Directive 86/609/EEC and the GlaxoSmithKline Policy on the Care, Welfare and Treatment of Animals.
Additional methods are described in Supplemental Methods online.

\section{Author contributions}

MP, YS, ASIL, and DWR conceived the project. MP, JEG, ASIL, and DWR designed the experiments, and MP, ASIL, and DWR wrote the paper. JEG performed some in vivo and ex vivo experiments in REV-ERB $\alpha$-knockout mice, and MP performed the majority of the experiments and downstream analyses. TWH and S. Brown performed the bone marrow transplant study, and TWH participated in some of the in vivo experiments. NB managed the transgenic animal colonies and provided technical help with in vivo experiments. $\mathrm{RV}$ performed the isolation of the bronchial cells by laser capture, lung section bioluminescence recordings, and technical help with in vivo experiments. TP and BG contributed to the work on posttranslational modification and degradation of REV-ERB $\alpha$. BS completed studies of in situ hybridization. DHJ, JWP, JPT, S. Bresciani, NCOT, AWJC, RPT, DG, WZ, and TMW performed all the chemistry around GSK1362 and its characterization. DAD led on the development of the antibody GSK6F05. ASM supervised the bone marrow transplant study. BB and PLP undertook the cigarette smoke studies.

\section{Acknowledgments}

We thank J. Woodburn for technical help; P. John-Baptiste, GlaxoSmithKline, for help with breeding the Rev-Erbo-knockout mice; and D. Carpenter, GlaxoSmithKline, for his work on flow cytometry analysis. We also thank S. Farrow for his contribution to this project. The work was supported by research grants awarded to DWR and ASIL from the Biotechnology and Biological Sciences Research Council, UK (BB/L000954/1), and an Engineering and Physical Sciences Research Council UK grant awarded to NCOT. DWR and ASIL are Wellcome investigators (107849/Z/15/Z). MP was funded by a Medical Research Council studentship jointly with a GlaxoSmithKline CASE PhD Scholarship.

Address correspondence to: David W. Ray or Andrew Loudon, Centre for Endocrinology and Diabetes, AV Hill Building, Oxford Road, Manchester M13 9PT, United Kingdom. Phone: 44.0.161.275.5655; Email: david.w.ray@manchester.ac.uk (D.W. Ray); andrew.loudon@manchester.ac.uk (A. Loudon).

BG's present address is: School of Healthcare Science, Manchester Metropolitan University, Manchester, United Kingdom.
1. Hussell T, Bell TJ. Alveolar macrophages: plasticity in a tissue-specific context. Nat Rev Immunol. 2014;14(2):81-93.

2. Gibbs J, et al. An epithelial circadian clock controls pulmonary inflammation and glucocorticoid action. Nat Med. 2014;20(8):919-926.

3. Mohawk JA, Green CB, Takahashi JS. Central and peripheral circadian clocks in mammals. Annu Rev Neurosci. 2012;35:445-462.

4. Bellet MM, et al. Circadian clock regulates the host response to Salmonella. Proc Natl Acad Sci U S A. 2013;110(24):9897-9902.

5. Gibbs JE, et al. The nuclear receptor REV-ERB $\alpha$ mediates circadian regulation of innate $\mathrm{immu}$ nity through selective regulation of inflammatory cytokines. Proc Natl Acad Sci U S A.
2012;109(2):582-587.

6. Narasimamurthy R, Hatori M, Nayak SK, Liu F, Panda S, Verma IM. Circadian clock protein cryptochrome regulates the expression of proinflammatory cytokines. Proc Natl Acad Sci U S A. 2012;109(31):12662-12667.

7. Scheiermann C, Kunisaki Y, Frenette PS. Circadian control of the immune system. Nat Rev Immunol. 2013;13(3):190-198.

8. Man K, Loudon A, Chawla A. Immunity around the clock. Science. 2016;354(6315):999-1003.

9. Spengler ML, et al. Core circadian protein CLOCK is a positive regulator of NF- $\mathrm{KB}$ mediated transcription. Proc Natl Acad Sci U S A. 2012;109(37):E2457-E2465.

10. Curtis AM, et al. Circadian control of innate immunity in macrophages by miR-155 targeting Bmal1. Proc Natl Acad Sci U S A. 2015;112(23):7231-7236.

11. Sato S, et al. A circadian clock gene, Rev-erb $\alpha$, modulates the inflammatory function of macrophages through the negative regulation of $\mathrm{Ccl} 2$ expression. JImmunol. 2014;192(1):407-417.

12. Lam MT, et al. Rev-Erbs repress macrophage gene expression by inhibiting enhancer-directed transcription. Nature. 2013;498(7455):511-515.

13. Cho H, et al. Regulation of circadian behaviour and metabolism by REV-ERB- $\alpha$ and REV-ERB- $\beta$. Nature. 2012;485(7396):123-127.

14. Zhang Y, et al. Discrete functions of nuclear receptor Rev-erb $\alpha$ couple metabolism to the clock. Science. 2015;348(6242):1488-1492. 
15. Trump RP, et al. Optimized chemical probes for REV-ERB $\alpha$. . J Med Chem. 2013;56(11):4729-4737.

16. Preitner N, et al. The orphan nuclear receptor REV-ERBalpha controls circadian transcription within the positive limb of the mammalian circadian oscillator. Cell. 2002;110(2):251-260.

17. Solt LA, et al. Regulation of circadian behaviour and metabolism by synthetic REV-ERB agonists. Nature. 2012;485(7396):62-68.

18. Jager J, et al. The nuclear receptor Rev-erb $\alpha$ regulates adipose tissue-specific FGF21 signaling. J Biol Chem. 2016;291(20):10867-10875.

19. Jeyaseelan S, et al. Induction of CXCL5 during inflammation in the rodent lung involves activation of alveolar epithelium. Am J Respir Cell Mol Biol. 2005;32(6):531-539.

20. Jacobsen EA, et al. Allergic pulmonary inflammation in mice is dependent on eosinophilinduced recruitment of effector T cells. J Exp Med. 2008;205(3):699-710.

21. Beck-Schimmer B, Schwendener R, Pasch T, Reyes L, Booy C, Schimmer RC. Alveolar macrophages regulate neutrophil recruitment in endotoxin-induced lung injury. Respir Res. 2005;6:61.

22. Elizur A, Adair-Kirk TL, Kelley DG, Griffin GL, deMello DE, Senior RM. Clara cells impact the pulmonary innate immune response to LPS. Am J Physiol Lung Cell Mol Physiol. 2007;293(2):L383-L392.

23. Bugge A, et al. Rev-erb $\alpha$ and Rev-erb $\beta$ coordinately protect the circadian clock and normal metabolic function. Genes Dev. 2012;26(7):657-667.

24. Eichenfield DZ, et al. Tissue damage drives co-localization of NF-kB, Smad3, and Nrf2 to direct Rev-erb sensitive wound repair in mouse macrophages. Elife. 2016;5:e13024.

25. Martinez Molina D, et al. Monitoring drug target engagement in cells and tissues using the cellular thermal shift assay. Science. 2013;341(6141):84-87.

26. Phelan CA, et al. Structural analysis of Rev-erb $\alpha$ bound to NCoR reveals a unique mechanism of nuclear receptor-corepressor interaction. Nat Struct Mol Biol. 2010;17(7):808-814.

27. Kojetin D, Wang Y, Kamenecka TM, Burris TP. Identification of SR8278, a synthetic antagonist of the nuclear heme receptor REV-ERB. ACS Chem Biol. 2011;6(2):131-134.

28. Arjona A, Silver AC, Walker WE, Fikrig E. Immunity's fourth dimension: approaching the circadian-immune connection. Trends Immunol. 2012;33(12):607-612.

29. Okada K, et al. Injection of LPS causes transient suppression of biological clock genes in rats. JSurg Res. 2008;145(1):5-12.

30. Vasu VT, Cross CE, Gohil K. Nr1d1, an important circadian pathway regulatory gene, is suppressed by cigarette smoke in murine lungs. Integr Cancer Ther. 2009;8(4):321-328.

31. Zhao X, et al. Circadian amplitude regulation via FBXW7-targeted REV-ERB $\alpha$ degradation. Cell. 2016;165(7):1644-1657.

32. Curtis AM, Bellet MM, Sassone-Corsi P, O'Neill LA. Circadian clock proteins and immunity. Immunity. 2014;40(2):178-186.

33. Yang $\mathrm{G}$, et al. Timing of expression of the core clock gene Bmal1 influences its effects on aging and survival. Sci Transl Med. 2016;8(324):324ra16.
34. Pourcet B, et al. Nuclear receptor subfamily 1 group $\mathrm{D}$ member 1 regulates circadian activity of NLRP3 inflammasome to reduce the severity of fulminant hepatitis in mice [published online ahead of print December 24, 2017] Gastroenterology. https://doi.org/10.1053/ j.gastro.2017.12.019.

35. Sulli G, et al. Pharmacological activation of REVERBs is lethal in cancer and oncogene-induced senescence. Nature. 2018;553(7688):351-355.

36. Li H, Cho SN, Evans CM, Dickey BF, Jeong JW, DeMayo FJ. Cre-mediated recombination in mouse Clara cells. Genesis. 2008;46(6):300-307.

37. Yoo SH, et al. PERIOD2::LUCIFERASE realtime reporting of circadian dynamics reveals persistent circadian oscillations in mouse peripheral tissues. Proc Natl Acad Sci U S A. 2004;101(15):5339-5346.

38. Podolin PL, et al. T cell depletion protects against alveolar destruction due to chronic cigarette smoke exposure in mice. Am J Physiol Lung Cell Mol Physiol. 2013;304(5):L312-L323.

39. Gibbs JE, et al. Circadian timing in the lung: a specific role for bronchiolar epithelial cells. Endocrinology. 2009;150(1):268-276.

40. Guilding C, Scott F, Bechtold DA, Brown TM, Wegner S, Piggins HD. Suppressed cellular oscillations in after-hours mutant mice are associated with enhanced circadian phase-resetting. JPhysiol (Lond). 2013;591(4):1063-1080.

41. Longo PA, Kavran JM, Kim M-S, Leahy DJ. Transient mammalian cell transfection with polyethylenimine (PEI). Methods Enzymol. 2013;529:227-240. 